\title{
Mechanical heterogeneity along single cell-cell junctions is driven by lateral clustering of cadherins during vertebrate axis elongation
}

Robert J. Huebner ${ }^{1 *}$, Abdul Naseer Malmi-Kakkada²,3*, Sena Sarikaya1, Shinuo Weng ${ }^{1}$, D. Thirumalai $^{\star \star 2}$, and John B. Wallingford ${ }^{\star \star 1}$

1. Department of Molecular Biosciences

2. Department of Chemistry, University of Texas at Austin.

3. Dept. of Chemistry and Physics, Augusta University

${ }^{\star}$ These authors contributed equally

${ }^{\star *}$ Co-corresponding authors:

John Wallingford

Patterson Labs

2401 Speedway

Austin, Texas

78712

Wallingford@austin.utexas.edu

512-232-2784

Dave Thirumalai

dave.thirumalai@gmail.com 


\begin{abstract}
:
Morphogenesis is governed by the interplay of molecular signals and mechanical forces across multiple length scales. The last decade has seen tremendous advances in our understanding of the dynamics of protein localization and turnover at sub-cellular length scales, and at the other end of the spectrum, of mechanics at tissue-level length scales. Integrating the two remains a challenge, however, because we lack a detailed understanding of the subcellular patterns of mechanical properties of cells within tissues. Here, in the context of the elongating body axis of a vertebrate embryo, we combine tools from cell biology and physics to demonstrate that individual cell-cell junctions display finely-patterned local mechanical heterogeneity along their length. We show that such local mechanical patterning is essential for the cell movements of convergent extension and is imparted by locally patterned clustering of a classical cadherin. Finally, the patterning of cadherins and thus local mechanics along cell-cell junctions are controlled by Planar Cell Polarity signaling, a key genetic module for CE that is mutated in diverse human birth defects.
\end{abstract}

\title{
Introduction:
}

The establishment and maintenance of animal form involves the control of physical forces by molecular systems encoded in the genome, and the elongation of an animal's head-to-tail body axis is a long-studied paradigm for understanding morphogenesis (Guillot and Lecuit, 2013). This essential step in the construction of a new embryo is driven by an array of morphogenetic engines, including an evolutionarily ancient suite of collective cell behaviors termed convergent extension (Fig. 1A; Supp. Fig. 1A)(Huebner and Wallingford, 2018). Critically, failure of axis elongation does not simply result in a shorter embryo, but rather has catastrophic consequences, and defects in convergent extension in mammals, including humans, results in lethal birth defects (Wallingford et al., 2013).

The biomechanics of convergent extension (CE) and axis elongation more generally have been studied across diverse length scales, providing several key insights (Davidson, 2017; Mongera et al., 2019; Stooke-Vaughan and Campàs, 2018). At the tissue scale, these include quantitative descriptions of patterned macroscopic stiffening (Moore et al., 1995; Zhou et al., 2009), tissuescale jamming transitions (Mongera et al., 2018; Serwane et al., 2017), and fluid-like multicellular flows (Bénazéraf et al., 2010; Lawton et al., 2013). At smaller length scales, laser ablation studies have provided insights into the patterns of junctional tension within these tissues (Fernandez-Gonzalez et al., 2009; Rauzi et al., 2008; Shindo and Wallingford, 2014). Finally, the mechanics of cell collectives have also been thoroughly explored using theoretical models (Alt et al., 2017; Fletcher et al., 2017; Merkel and Manning, 2017; Staddon et al., 2019).

Despite these advances, we know comparatively little about sub-cellular mechanical properties in vivo or their molecular basis. Resolving this disconnect is crucial, because CE in diverse systems is known to require complex spatial and temporal patterns of protein localization and 
dynamics along individual cell-cell junctions during morphogenesis. For example, the localization and turnover of actomyosin and cadherin adhesion proteins have been extensively quantified during Drosophila CE (Blankenship et al., 2006; Fernandez-Gonzalez et al., 2009; Levayer and Lecuit, 2013; Rauzi et al., 2008)), as have similar patterns for the Planar Cell Polarity (PCP) proteins and actomyosin during vertebrate CE (Butler and Wallingford, 2018; Kim and Davidson, 2011; Shindo and Wallingford, 2014). However, the significance of these molecular patterns remains unclear because we lack a similarly granular understanding of subcellular mechanical properties and their dynamics, which ultimately explain the cell behaviors that drive CE.

Here, we combine high-speed super-resolution microscopy with concepts rooted in soft matter physics to demonstrate that individual cell-cell junctions in the elongating vertebrate body axis display finely-patterned local mechanical heterogeneity along their length. To explore this unexpected finding, we developed a new theory for junction remodeling in silico and new tools for assessment of very local mechanics in vivo. Combining these, we show that sub-cellular mechanical heterogeneity is essential for CE and is imparted by cadherins via locally patterned intracellular (cis-) interactions. Finally, the local patterns of both cadherin clustering and heterogeneous junction mechanics are controlled by PCP signaling, a key regulatory module for CE that is mutated in diverse human birth defects.

\section{Results:}

\section{The dynamics of tricellular junction motion during CE suggest an unexpected mechanical heterogeneity at subcellular length scales}

The elongating body axis of Xenopus embryos is a long-standing and powerful model system for studying PCP-dependent vertebrate CE (Supp. Fig. 1)(Huebner and Wallingford, 2018). Xenopus CE can be considered most simply in terms of four-cell neighbor exchanges in which mediolaterally-aligned cell-cell junctions ("v-junctions") shorten, followed by the elongation of new, perpendicularly-aligned junctions ("t-junctions")(Fig. 1A). To gain deeper insights into this process, we used high-speed super-resolution imaging to establish a quantitative physical description of the motion of tricellular vertices bounding v-junctions (Fig. 1B).

We found that v-junction shortening was dominated by the movement of a single "active" vertex, while the other "passive" vertex moved comparatively less (Fig. 1C, D)(SI, Section 1). Three distinct metrics demonstrated that this asymmetry was not a point-of-reference artifact (Supp. Fig. 2). Such asymmetry has also been observed during Drosophila CE (Vanderleest et al., 2018) so may represent a fundamental feature. We therefore explored its physical basis using mean squared displacement (MSD), which reveals physical properties of motion (SI Section 2).

Our analysis revealed that active vertices consistently displayed a highly fluidized movement (i.e. super-diffusive)(Fig. 1E, F, red). By contrast, passive vertices displayed the hallmarks of more-constrained, glass-like motion (i.e. defined by sub-diffusive movement with an intermediate time slowdown, as observed in colloidal systems; (Kegel and van Blaaderen, 2000; Weeks et al., 2000) (Fig. 1E, F, blue). The juxtaposition of liquid- and glass-like motion along a single cell-cell junction was interesting, because while fluid-to-glass phase transitions are known features at tissue-level length scales (Angelini et al., 2011; Bi et al., 2015; Malmi-Kakkada et al., 
2018; Sinha et al., 2020), such transitions have not been reported at sub-cellular length scales during morphogenesis. To confirm this surprising result, we applied four additional physical metrics, the Van Hove function, the velocity auto-correlation function, the self-overlap parameter, and the fourth order susceptibility, $\chi_{4}(t)$ (SI sections 2-4).

All four orthogonal approaches confirmed our finding that the active and passive vertices bounding individual v-junctions exhibit asymmetric dynamic behaviors, with one vertex displaying a fluid-like motion and the other, glass-like (Supp. Fig. 2, 3). Critically, this asymmetric behavior was specific to shortening dynamics of v-junctions, as the two vertices bounding non-shortening junctions in the same tissue were consistently symmetrical, both resembling passive vertices (Fig. 1E, F, pink, black; Supp. Fig. 2, 3).

This physical analysis provided three important insights: First, glass-like dynamics previously observed only at tissue-length scales in morphogenesis also exist at the sub-cellular length scale of individual junctions. Second, the frequently invoked assumption of mechanical homogeneity along single cell-cell junctions, which underlies a wide swath of the biophysical work on morphogenesis, may not be valid. And finally, because only shortening junctions exhibited local mechanical heterogeneities, this phenomenon may be a specific and essential feature of convergent extension.

\section{A new physical model of cell-cell junction remodeling predicts heterogeneous mechanics along single junctions is an essential feature of convergent extension}

The possibility of mechanical heterogeneity along single cell-cell junctions has important implications, as many biophysical approaches and in silico tools for understanding morphogenesis (e.g. laser cutting, vertex models) assume that junctions are mechanically homogeneous. We therefore developed a new theoretical framework for junction shortening that accommodates the possibility of local mechanical heterogeneity by independently modeling the movement of each vertex (Fig. 2A, B)(SI, Section 5-9).

Our model involves (i) a local junction stiffness (or elasticity) modeled using a spring element, which is consistent with the pulsatile relaxation of v-junctions observed in Xenopus CE (Shindo and Wallingford, 2014); (ii) a dynamic rest length, recently shown to be important for modeling CE (Staddon et al., 2019); (iii) a viscoelastic parameter, $k / \gamma$, dictated by the spring stiffness, $k$, and the friction at the vertices, $\gamma$; and (iv) a rest length exponent, $\psi$, which describes the time dependence of plastic displacement of the vertices modeled with a piston (Fig. 2A, B)(SI, Section 5-7).

Using this model, we explored parameter space to find variables in elastic and viscous deformation that can support effective shortening of the junction (SI, Sections 8, 9). As shown in the heatmap in Fig $\mathbf{2 C}$, for a given rest length exponent, junctions failed to shorten if the viscoelastic parameter was equal and small for both the vertices (Fig. 2C, red box)(SI, Sections 8, 9). When the viscoelastic parameter was asymmetric, junctions shortened effectively (Fig. 2C, gold box). Thus, at the level of binary outcome (i.e. shorten versus fail-toshorten), our model suggests that CE requires mechanical heterogeneity along single vjunctions. 
For a more stringent test, we compared the temporal dynamics of junction shortening in our model to those quantified in vivo from high-speed super-resolution movies (Fig. 2D, E). In timelapse data, the relaxation behavior of v-junctions collapsed into a self-similar pattern when normalized; relaxation became progressively more efficient over time and could be described by a compressed exponential (Fig. 2G, black lines; Supp. Fig. 4A, B)(SI, Sections 10, 11). When the viscoelastic parameters in our model were asymmetric, the shortening dynamics closely recapitulated this compressed exponential relaxation (Fig. 2G, gray lines, I; expanded view in Supp. Fig. 4C).

Finally, we also analyzed junction length dynamics in non-shortening junctions in vivo (Fig. 2D, F), which we found above to display symmetrical mechanics along their length (Fig. 1E, black, pink). The length dynamics of non-shortening junctions in vivo displayed wide fluctuations over time, did not share a self-similar relaxation pattern, and displayed large deviations from the compressed exponential (Fig. 2H, black, I). Strikingly, when symmetrical parameters for $k / \gamma$ were input into the model, the resulting junction length dynamics displayed wide fluctuations in length and deviated substantially from the normal relaxation pattern (Fig. 2H, gray; I).

Thus, by incorporating local mechanical heterogeneity, our new model not only recapitulates overall shortening/non-shortening outcomes, but also quantitatively recapitulates the dynamic patterns of length change observed in both shortening and non-shortening junctions in vivo. Because both modeling and observations suggest a key role for mechanical heterogeneity, we next sought to understand the contribution of such local mechanical regimes to cell movement during CE.

\section{Fluid-like directed motion of active vertices results from restriction of transverse fluctuations in motion}

Our theory makes a curiously counter-intuitive prediction: that the more fluid-like motion of the active vertex occurs in the context of increased local stiffness (i.e. higher viscoelastic parameter), while the more glass-like motion of the passive vertex occurs in a relatively decreased stiffness regime. To address this seeming paradox, we considered that vertex movement, while highly directional, is not entirely directed (Fig. 3A). We then reasoned that a stiffer mechanical regime might limit transverse fluctuations near active vertices (Fig. 3A, green), thereby resulting in more smoothly processive, fluid-like motion in the line of shortening as compared to passive vertices (Fig. 3A, orange).

To test our model's prediction in vivo, we used our time-lapse data to quantify the transverse fluctuations of vertices (Fig. 3B, green arrows)(SI, Section 16). Consistent with our model's prediction, active vertices displayed significantly less transverse fluctuation than did passive vertices at the same junctions (Fig. 3B, C, E), indicating a higher local stiffness at active vertices (Marmottant et al., 2009). Analysis of the straightness index independently validated this conclusion (Fig. 3E, F)(SI, Section 16).

This analysis of in vivo imaging data validates our physical model's prediction of an increased stiffness regime near active junctions and suggests that the lower stiffness regime of passive vertices allows more transverse fluctuation, resulting in less-directed, more glass-like movement. As such, multiple independent lines of observation and theory suggest that local mechanical heterogeneity along cell-cell junctions is a fundamental feature of CE. We next 
sought to understand the molecular underpinnings of this feature, asking if patterns of protein localization during CE might reflect the local mechanical patterns we identified here.

\section{Patterned cis-clustering of cadherins reflects the heterogeneous mechanics along shortening junctions}

We turned our attention to cadherin cell adhesion proteins, and specifically Cdh3, which is essential for CE in Xenopus (Brieher and Gumbiner, 1994; Fagotto et al., 2013)(Supp. Fig. 5A) and was recently implicated in CE cell movements in the mouse skin (Cetera et al., 2018). Like all classical cadherins, Cdh3 forms both intercellular trans-dimers and also cis-clusters mediated by intracellular interactions (Fig. 4A). Cis-clustering is a key regulatory nexus for cadherin function (Yap et al., 2015), so it is interesting that while the mechanisms governing formation of cadherin cis-clusters during CE has been studied, cis-cluster function during CE remains unknown (e.g. (Levayer and Lecuit, 2013; Truong Quang et al., 2013).

We used high-speed super-resolution microscopy to image a functional GFP-fusion to Cdh3 and used the spatial autocorrelation function for an unbiased quantification of Cdh3-GFP cluster size (SI, Section 15). Using this function, an exponential decay in spatial correlation is expected for clusters that are regularly ordered, and this pattern was observed for Cdh3-GFP (Fig.4C). Moreover, this decay reached zero at $\sim 1 \mu \mathrm{m}$ (Fig. 4C), consistent with the size reported for cisclusters of cadherins in vertebrate cell culture (Yap et al., 2015).

This analysis also revealed that Cdh3 clusters undergo dynamic fluctuations in size (Fig. 4D). Moreover, junction shortening is pulsatile (Fig. 4E)(Shindo and Wallingford, 2014) and fluctuations in mean Cdh3 cluster size significantly cross-correlated with shortening pulses (Fig. 4D). Mean cluster size peaked $\sim 20$ seconds prior to the onset of junction shortening pulses (Fig. 4E, F)(SI, Section 16), suggesting a functional relationship between Cdh3 clustering and junction remodeling.

We then reasoned that mechanical heterogeneity observed along cell-cell junctions during CE might be driven by local patterns of Cdh3 clustering, since cadherins can tune the local mechanics of free cell membranes in single cultured cells (Strale et al., 2015). This led us to measure Cdh3 cluster size specifically in the 3-micron region abutting vertices of shortening vjunctions during shortening pulses. Patterns of Cdh3 clustering were complex and highly heterogeneous, consistent with the mechanical heterogeneities we report here (Supp. Fig. 6). Nonetheless, the mean size of Cdh3 clusters near active junctions was significantly larger than that for clusters near passive vertices (Fig. 4G). We confirmed this important result using an alternative quantification of cluster size involving fits to the exponential decay of the spatial autocorrelation (Supp. Fig.5 C,D)(SI, Section 17).

Importantly, asymmetric Cdh3 clustering was specific to shortening v-junctions and was not observed along non-shortening junctions in the same tissue. Rather, all vertices bounding nonshortening junctions displayed clustering similar to that near passive vertices in shortening junctions (Fig. 4G). Symmetrical clustering in non-shortening reflects the symmetrical dynamics of vertices bounding these junctions, described above (Fig. 1E). Accordingly, these results demonstrate that asymmetric cis-clustering of Cdh3 is a specific property of shortening vjunctions during $\mathrm{CE}$ and suggests that such clustering may drive the asymmetric mechanics of active and passive vertices that we observed in vivo and predicted in silico. 


\section{Cdh3 cis-clustering is required for axis elongation but not homeostatic tissue cohesion in vivo.}

The patterned, asymmetric cis-clustering of Cdh3 during CE is a significant finding, because as mentioned above the function of cis-clustering remains undefined not only for CE, but indeed in any in vivo context. We therefore took advantage of point mutations in Cdh3 that specifically disrupt the hydrophobic pocket that mediates cis clustering, without affecting trans dimerization (cisMut-Cdh3; Fig. 5A)(Harrison et al., 2011; Strale et al., 2015). To test this mutant in vivo, we depleted endogenous Cdh3 as previously described (Supp. Fig. 7)(Ninomiya et al., 2012), and then re-expressed either wild-type Cdh3-GFP or cisMutant-Cdh3-GFP.

We first confirmed the cis mutant's impact on clustering in vivo. Re-expressed wild-type Cdh3GFP clustered normally and displayed the expected exponential decay in spatial autocorrelation that indicates regular spatial order and a mean cluster size $\sim 1 \mu \mathrm{m}$ (Fig. 5B, D)(SI, Section 1618). By contrast, when cisMut-Cdh3-GFP was re-expressed, clusters were clearly absent, and the signal was diffuse along cell-cell junctions (Fig. 5C). Moreover, the spatial autocorrelation of cisMut-Cdh3-GFP did not decay exponentially (Fig. 5D), consistent with a lack of spatial order (SI, Section 16-18). We confirmed this result using fits to the exponential decay of the spatial autocorrelation (Supp. Fig. 8B-G)(SI, Section 16-18).

We next used the same replacement strategy to directly test the function of cis-clustering in Xenopus CE. At neurulation stages, embryos depleted of Cdh3 display severe defects in axis elongation (Fig. 5E, F, I, green)(Brieher and Gumbiner, 1994; Lee and Gumbiner, 1995). At later stages, these embryos disassociate to individual cells due to the widespread requirement for Cdh3 in cell cohesion (Ninomiya et al., 2012)(Fig. 5J, green). We found that re-expression of wild-type Cdh3-GFP rescued both axis elongation and embryo cohesion, as expected (Fig. $5 G$, I, J, purple).

Strikingly however, while re-expression of cisMut-Cdh3-GFP significantly rescued overall embryo cohesion (Fig. 5J, red), it failed to rescue axis elongation (Fig. $\mathbf{5 H}$, I, red). These data provide the first experimental test of the role of cadherin cis-clustering in vivo, and moreover, provide an experimental entry point for testing the role of cis-clustering in the generation of local mechanical patterns along cell-cell junctions.

\section{Loss of Cdh3 cis-clustering eliminates mechanical heterogeneity and disrupts shortening dynamics of cell-cell junctions during $C E$.}

To understand the relationship between Cdh3 clustering (Fig. 4) and the asymmetric mechanics and vertex dynamics of shortening v-junctions (Figs. 1-3), we applied our battery of physical methods to quantify the motion of vertices in cells with disrupted Cdh3 cis-clustering (i.e. Cdh3 knockdown + cisMut-Cdh3 re-expression). We found first that defects in axis elongation in cisMut-Cdh3 expressing cells were accompanied by defects in cell polarization (Fig. 6A-C), reflecting the phenotype seen when PCP signaling is disrupted (Wallingford et al., 2000). Second, v-junctions in cells with disrupted Cdh3 clustering displayed large fluctuations in length that deviated significantly from the compressed exponential relaxation pattern observed for normal v-junctions (Fig. 6D, E). The aberrant length dynamics of cisMut-Cdh3 expressing junctions resembled those of junctions that lack mechanical heterogeneity (i.e. non-shortening junctions in normal embryos in vivo or those modeled in silico (compare Fig. 6D with Fig. 2H). 
We then asked if cisMut-Cdh3 expression also disrupted the normal mechanical heterogeneity of v-junctions by quantifying transverse fluctuations of vertices. We found that all vertices in cells with defective cis-clustering of Cdh3 displayed the elevated transverse fluctuations observed only in passive vertices of normal cells (Fig. 6F, G). These results provide direct experimental evidence that Cdh3 cis-clustering restricts transverse movement of vertices, thereby facilitating fluid-like shortening of the junction.

\section{PCP is essential for Cdh3 cis-clustering and mechanical heterogeneity at cell-cell junctions.}

A key challenge in animal morphogenesis is to understand how ubiquitous cellular machinery such as cadherin adhesion is directed by tissue-specific developmental control mechanisms. PCP signaling is a central regulator of vertebrate CE and PCP proteins localize to shortening vjunctions during Xenopus CE, where they control actomyosin contractility (Butler and Wallingford, 2018; Shindo et al., 2019), but how these systems interface with cadherin adhesion during $\mathrm{CE}$ is poorly defined. Because cells with disrupted Cdh3 cis-clustering superficially resemble those with defective PCP (Fig. 6B, C), we asked if Cdh3 clustering may be under the control of PCP signaling.

We disrupted PCP with the well-characterized dominant-negative version of Dvl2, Xdd1, which severely disrupted cell intercalation behaviors as expected (Wallingford et al., 2000)(Fig. 6C). Strikingly, expression of Xdd1 also elicited a significant disruption of Cdh3 clustering that was apparent in both images and in the lack of exponential decay in spatial autocorrelation data (Fig. 7B, C). Finally, Xdd1 expressing junctions also displayed exaggerated length fluctuations, significant deviation from the compressed exponential relaxation behavior, and symmetrical, elevated transverse fluctuations (Fig. 7D-F), all features associated only with junctions lacking local mechanical heterogeneity in vivo or in silico.

These data not only provide an independent experimental confirmation of the link between Cdh3 cis-clustering, local mechanical heterogeneity of junctions, and asymmetric vertex dynamics

(Fig. 7G), but also provide a novel mechanistic link between a conserved and essential developmental regulatory module (PCP), and the ubiquitous machinery of Cadherin adhesion.

\section{Discussion:}

Here, we combined physical and cell biological approaches to observation, theory, and experiment to identify and link two novel features of vertebrate convergent extension, one physical, the other molecular. First, we show that single cell-cell junctions in vivo display patterned mechanical heterogeneities along their length. Second, we show that locally patterned cis-clustering of a classical cadherin impart these patterns of mechanical heterogeneity under the control of PCP signaling.

These results are fundamentally important, because mechanical homeostasis in tissues is an emergent property of forces interacting across a wide range of length scales, yet we still know little about the sub-cellular mechanical properties of cells within tissues. Thus, while previous studies describe local heterogeneity in the membranes of single cultured cells (e.g. (Lieber et 
al., 2015; Shi et al., 2018; Strale et al., 2015)), our demonstration of local mechanical heterogeneity along single cell-cell junctions in an intact tissue is a substantial advance. Indeed, our data demonstrate that it is not the local heterogeneity per se, but rather its local patterning along individual cell-cell junctions that is a specific and essential feature of the junctional remodeling that drives CE. From a physical standpoint, this insight is important because it implies that the origin of patterned dynamic heterogeneities observed at tissue length scales (e.g. (Angelini et al., 2011; Bi et al., 2015; Malmi-Kakkada et al., 2018)) may reside in similarly complex patterns at length scales as small as that of individual cadherin clusters.

Our findings are also important for understanding the unifying suite of CE cell behaviors that is deeply conserved across evolution. V-junction shortening is accomplished by a combination of cell crawling via mediolaterally-positioned lamellipodia and active contraction of anteroposteriorly-positioned cell-cell junctions (Sun et al., 2017; Williams et al., 2014), a pattern that has now been described in animals ranging from nematodes, to insects to vertebrates (Huebner and Wallingford, 2018). While it remains to be determined whether v-junction shortening in other tissues and animals also displays the heterogeneity we report here, it is nonetheless remarkable that even subtle aspects (e.g. active and passive vertices) are similar in tissues as diverse as Drosophila epithelial cells (Vanderleest et al., 2018) and Xenopus mesenchymal cells (Fig. 1). Our data therefore suggest that this deeply evolutionarily conserved cell biological process may have a fundamental physical basis.

Finally, our findings also have important implications for cadherin biology. The lateral cisclustering of cadherins was first described decades ago (Yap et al., 1997) and has been extensively characterized using structural, biochemical, and cell biological approaches (Chen et al., 2015; Fagotto et al., 2013; Hong et al., 2013; Kale et al., 2018; Levayer and Lecuit, 2013; Levayer et al., 2011; Truong Quang et al., 2013; Yap et al., 1998). Strikingly, however, the functional consequences of defective cis-clustering in intact tissues have not been described, so our findings here fill a critical gap. Importantly, the essential role for patterned cis-clustering of Cdh3 is likely relevant far beyond the context of Xenopus axis elongation, as mouse Cdh3 (aka p-cadherin) is also implicated in PCP-mediated CE movements in the mouse skin (Cetera et al., 2018). Given that PCP genes are among the most well-defined genetic risk factors for human neural tube defects (Wallingford et al., 2013), our data provide insights that span from the fundamental physics of living cells, to the cell and developmental biology of vertebrate embryos, to the etiology of human birth defects.

\section{Acknowledgements:}

We thank Dan Dickinson for use of the iSIM microscope and for critical reading and helpful discussions. We thank Andy Ewald for critical reading of the manuscript. This work was supported by grants from the NICHD (R21HD084072) and the NIGMS (R01GM104853) to J.B.W. and from the N.S.F. (Phys 17-08128) and the Collie-Welch Chair through the Welch Foundation (F-0059) to D.T. 


\section{Supplemental Experimental methods}

\section{Xenopus embryo manipulations:}

Ovulation was induced by injection of adult female Xenopus with 600 units of human chorionic gonadotropin and animals were kept at $16^{\circ} \mathrm{C}$ overnight. Eggs were acquired the following day by squeezing the ovulating females and eggs were fertilized in vitro. Eggs were dejellied in $3 \%$ cysteine $(\mathrm{pH}$ 8) 1.5 hours after fertilization and embryos were reared in 1/3X Marc's modified Ringer's (MMR) solution. For microinjection, embryos were placed in $2 \%$ ficoll in 1/3X MMR and then washed in 1/3X MMR after injection. Embryos were injected using a Parker's Picospritizer III with an MK1 manipulator. Embryos were injected in the dorsal blastomeres at the 4 cells stage targeting the dorsal marginal zone. Keller explants were excised at stage 10.25 in Steinberg's solution using eyelash hair tools.

\section{Morpholino, plasmids, antibody, and cloning:}

The Cdh3 morpholino had been previously described (Ninomiya et al. JCS 2012) and was ordered from Gene Tools. Cdh3-GFP, (Pfister et al. Development 2016) lifeact-RFP, and membrane-BFP were made in pCS105 and Xdd1 was made in CS2myc (Sokol et al. Curr Biol., 1996). Cdh3 antibody was ordered from Developmental Studies Hybridoma Bank (catalog number 6B6). The Cdh3-cis-mutant was generated using the Q5 Site-Directed Mutagenesis Kit (NEB, catalog number A13282) and here we changed valine 259 to aspartic acid and isoleucine 353 to aspartic acid.

\section{Morpholino and mRNA microinjections:}

Capped mRNA was generated using the ThermoFisher SP6 mMessage mMachine kit (catalog number AM1340). mRNAs were injected at the following concentrations per blastomere, Membrane-BFP (100pg), Cdh3-GFP for imaging (50pg), Cdh3-GFP for rescue (300pg), Cdh3-cis-mutant (300pg), lifeact-RFP (100pg), and Xdd1 (1ng). Cdh3 morpholino was injected at a concentration of 10 ng per blastomere.

\section{Imaging Xenopus explants:}

Explants were mounted on fibronectin coated glass coverslips in either Steinberg's solution or Danilchik's for Amy solution. Experiments were repeated in the absence of fibronectin to ensure fibronectin did not confound results. Explants were then incubated at room temperature for 4 hours or at $16^{\circ} \mathrm{C}$ overnight before imaging. Standard confocal images were acquired with either a Nikon A1R or a Zeiss LSM 700. Super-resolution images were acquired with a commercially available instantaneous structured illumination microscope (BioVision Technologies).

\section{Measurement of Cdh3 intensity at cell junctions:}

All image analysis was performed using the open-source image analysis software Fiji (Schindelin et al. Nat. Methods 2012). Images were first processed with 50-pixel rolling ball radius background subtraction and smoothed with a $3 \times 3$-averaging filter, which allowed better distinction of individual cadherin clusters. The segmented line tool, with width set to the thickness of the junction ( 16 pixels), was used to set a line of interest (LOI) across the length of the cell junction. Next the multi-plot tool was used to extract cdh3 intensity values across the length of the cell and the measure tool was used to collect data such as junction length and mean intensity values. The Fiji Time Lapse plugin Line Interpolator Tool was used to make successive measurements for movies. Here a segmented line LOI was drawn every 10-30 frames, the line interpolator tool was then used to fill in the LOls between the manually drawn 
LOls allowing rapid semi-manual segmentation. The multi-plot tool and measure tool were then used to extract data for each time-point of the movie.

\section{Cdh3 immunostaining:}

Samples were prepared by micro-dissection as described above and incubated at room temperature for four hours or overnight at $16^{\circ} \mathrm{C}$. Samples were then fixed in 1x MEMFA for 1 hour at room temperature and washed 3 times with PBS to remove fixative. Next samples were permeabilized with $0.05 \%$ Triton X-100 in PBS for 30 minutes and then blocked in 1\% normal goat serum (NGS) in PBS for two hours at room temperature. The primary antibody was then diluted $1: 100$ in fresh $0.1 \%$ NGS/PBS and samples were incubated with primary antibody at $4{ }^{\circ} \mathrm{C}$ overnight. Samples were then blocked a second time at room temperature for 1 hour and then washed twice with fresh blocking solution. Secondary antibody (goat anti-Mouse 488, \#A32723) was diluted 1:500 and samples were incubated at $4^{\circ} \mathrm{C}$ overnight. Finally samples were washed three times in $1 \mathrm{X}$ PBS and imaged.

\section{Embryo length to width measurement:}

Embryos were injected in the dorsal blastomeres with Cdh3-MO, Cdh3-MO + Cdh3-GFP (rescue), Cdh3-MO + Cdh3-cis-mutant (mutant), or left as un-injected controls. Live embryos were kept at room temperature for 26 hours post fertilization ( stage 33$)$. Embryos were then fixed with MEMFA in glass vials on and rotated for 1 hour at room temperature. Post fixation samples were washed three times in $0.1 \%$ Tween-20 in 1X PBS and then images of embryos were acquired using a Zeiss AXIO Zoom stereoscope. The embryos anterior-posterior length and dorsal-ventral width were then measured using Fiji. Different conditions were statistically compared using a one-way non-parametric ANOVA (Kruskal-Wallis test).

\section{Embryo survivability assay:}

Embryos were injected in the same manner as done for the length to width measurement and the number of embryos injected for each condition was recorded. Embryos were then kept at room temperature for 20 hours ( stage 20$)$ and the number of surviving embryos was recorded. The percentage of embryos surviving was reported.

\section{Measurement of Cdh3 knockdown efficiency:}

Embryos were injected at the 4-cell stage in a single blastomere with Cdh3-MO + membrane-BFP generating embryos with mosaic knockdown of Cdh3 on one side of the embryo. Explants were next dissected from embryos, immuno-stained for $\mathrm{Cdh} 3$, and images were acquired as described above. The mosaic labeling allowed us to compare wild type and Cdh3-KD cells (marked by membrane-BFP) within a single explant. First we used Fiji to measure endogenous Cdh3 intensity at cell junctions in wild type and Cdh3-KD cells and used a t-test to statistically compare these conditions. Next cellular polarity was assessed for each condition by measuring the ratio of the mediolateral length to the anterior-posterior width of individual cells. A t-test was then used to show a significant reduction in cellular polarity in cells with Cdh3-KD. 
bioRxiv preprint doi: https://doi.org/10.1101/2020.02.11.944033; this version posted November 16, 2020. The copyright holder for this preprint (which was not certified by peer review) is the author/funder. All rights reserved. No reuse allowed without permission.

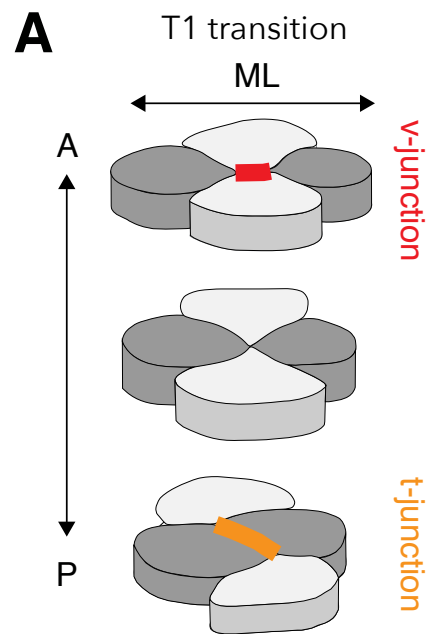

$\mathbf{E}$

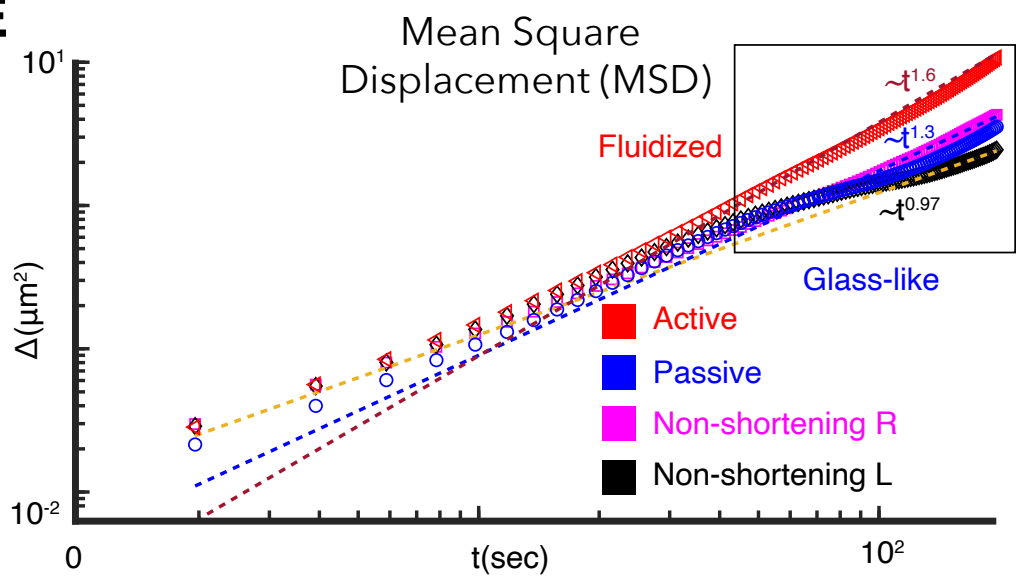

C

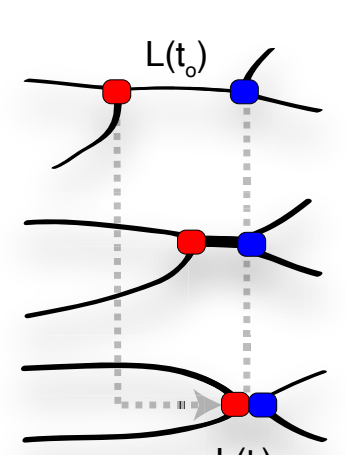

$L\left(t_{t}\right)$

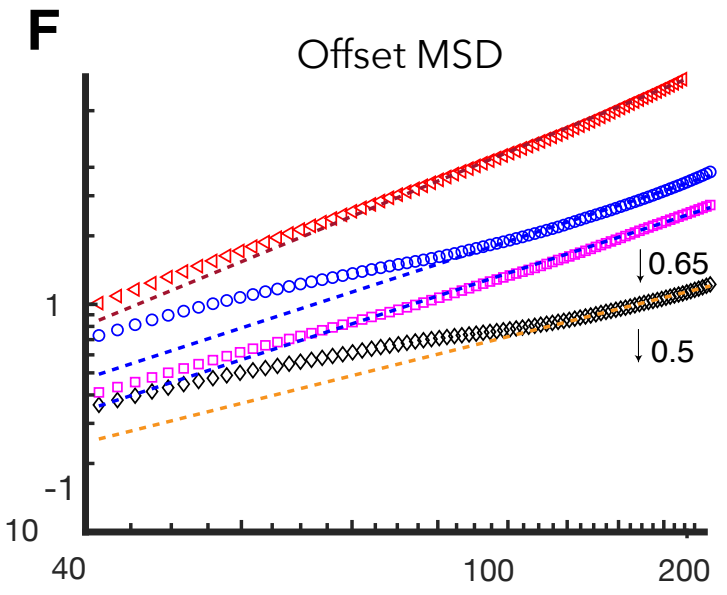

D Activity Parameter

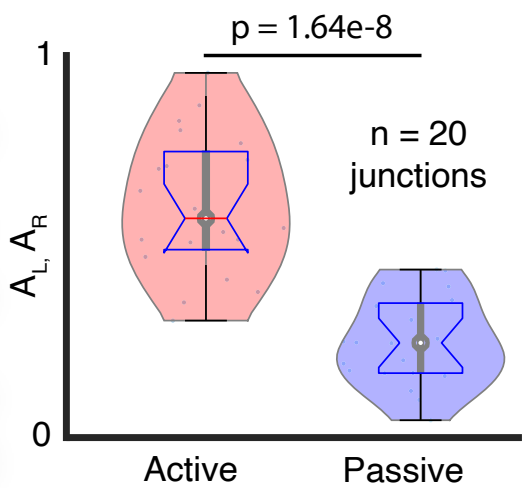

Active Passive 
Figure 1. Vertices bounding shortening v-junctions are physically asymmetric and display heterogeneous fluid and glass-like dynamics.

A. A four cell T1 transition with mediolaterally (ML)-aligned "v-junctions" (red) and anteriorposterior (A/P) aligned t-junctions (orange) indicated. B. Frames from time-lapse showing vertex movements of a v-junction; arrows highlight vertices. C. Schematic of asymmetric vertex movements from B; active = red; passive = blue. D. Vertex motion quantified by the activity parameter, as described in SI, Section 1. ( $N=42$ vertices from 20 embryos; t-test $p$ value is shown). E. MSD reveals active vertices' persistent superdiffusive movement (red); passive vertices exhibit intermediate time slowdown (blue). Pink and black display MSD for left and right non-shortening junctions. $F$. MSD from boxed region in $E$ is shown with traces offset for clarity ( 0.5 for left; 0.65 for right)( $\mathrm{N}=20$ vertices from 10 embryos). 
bioRxiv preprint doi: https://doi.org/10.1101/2020.02.11.944033; this version posted November 16, 2020. The copyright holder for this preprint (which was not certified by peer review) is the author/funder. All rights reserved. No reuse allowed without permission.

A

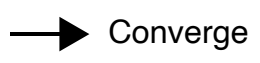

Active vertex Passive vertex

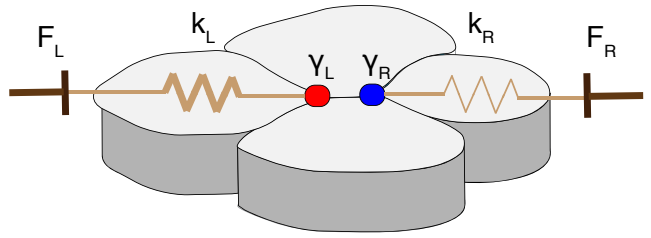

Actuator $=$ viscoplastic displacement Spring = elastic displacement
B $\frac{d x_{L}}{d t}=-\frac{k_{L} x_{L}}{\gamma_{L}}+\frac{F_{L}}{\gamma_{L}}+\zeta_{L}$ Passive vertex $\frac{d x_{R}}{d t}=-\frac{k_{R} x_{R}}{\gamma_{R}}+\frac{F_{R}}{\gamma_{R}}+\zeta_{R}$

$\mathrm{k}_{\mathrm{L}}=$ vertex elasticity $\mathrm{Y}_{\mathrm{L}}=$ Local viscosity $\zeta=$ Noise $\mathrm{F}_{\mathrm{L}}=\mathrm{k}_{\mathrm{L}} \mathrm{a}_{\mathrm{L}} \mathrm{t}_{\mathrm{L}}$ (Force for viscous deformation) $\psi_{\mathrm{L}}=$ Rest length exponent

\section{Phase Space of Theory Parameters}

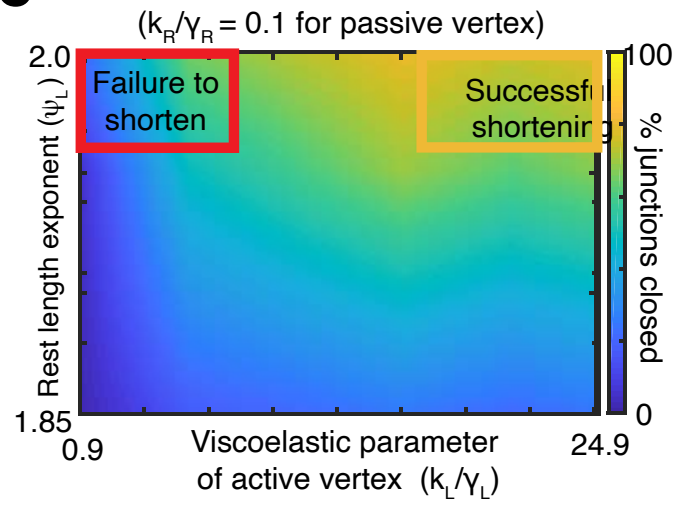

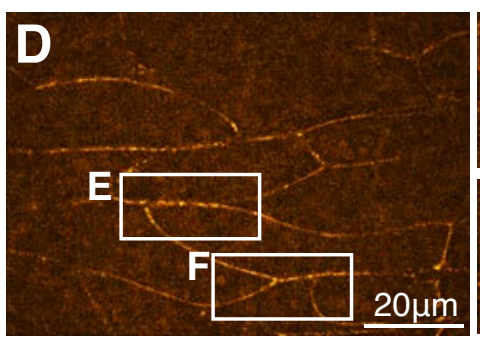

G Scaled Normalized

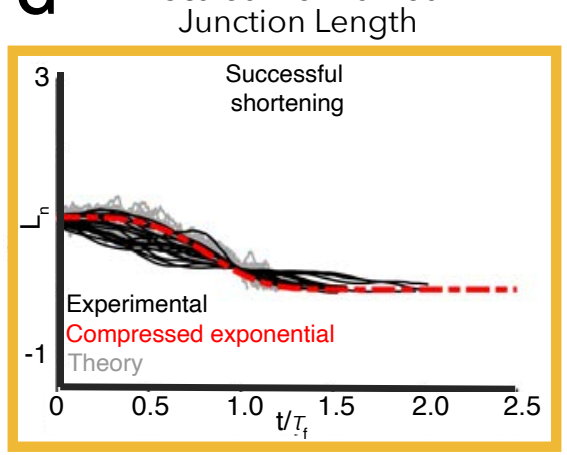

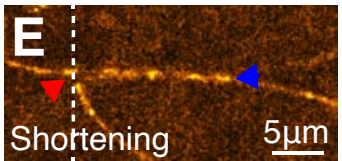
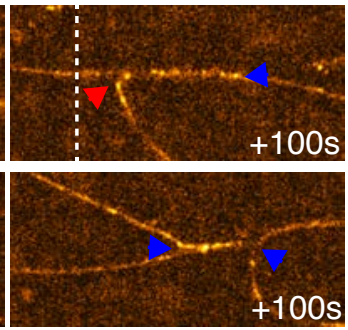
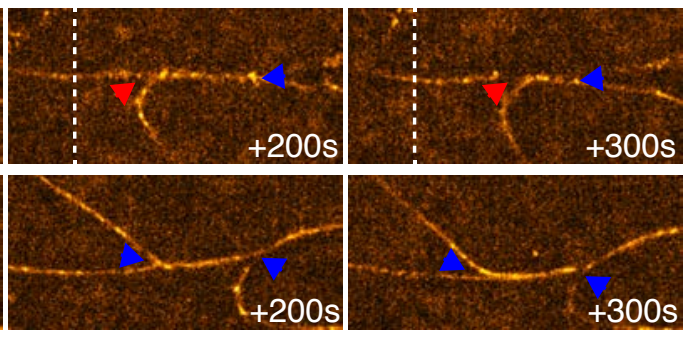

H Scaled Normalized

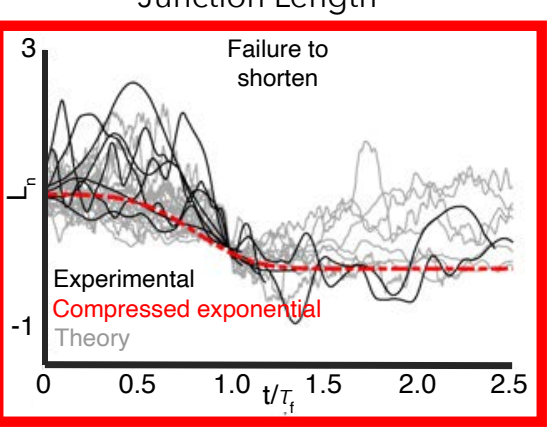

Deviation from compressed exponential

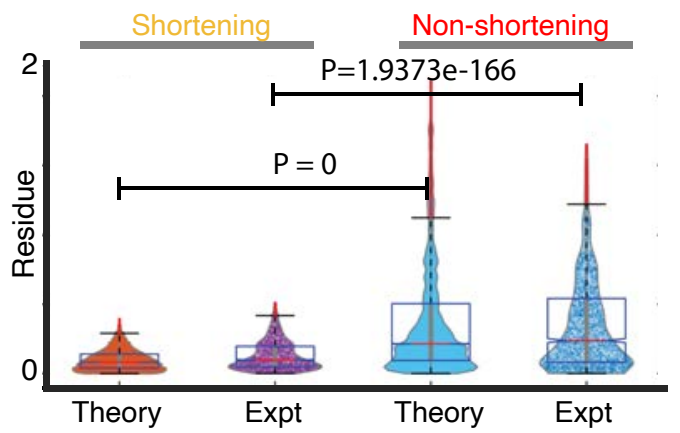


Figure 2. A new vertex model incorporating local mechanical heterogeneity recapitulates the fine-scale dynamics of junction shortening observed in vivo.

A. Sketch of v- junction shortening with elements of the model overlain. Active (red) and passive (blue) vertex movements are effected by a piston modulating the dynamic rest length. The vertices execute elastic motion due to springs of elasticity, $k_{L}$ and $k_{R} . L, R$ indices indicate left and right. The thicker spring indicates a stiffer elasticity constant, $k_{L}$. B. Equations of motion for active and passive vertex positions, $x_{L}$ and $x_{R}$. Displacement of the left (right) vertex due to the piston is determined by the forces $F_{L}\left(F_{R}\right)$ whose time dependence is determined by the rest length exponent, $\psi_{L}\left(\psi_{R}\right)$. The friction experienced by the left (right) vertices are modeled using $\gamma_{L}\left(\gamma_{R}\right) . \zeta_{L}$ is the colored noise term for the left vertex (SI, Section 5-7). C. Heatmap indicating probability of successful junction shortening (legend at right) in parameter space for the viscoelastic parameter near vertices and the rest length exponent, staying within biologically reasonable values based on data from Drosophila (Solon et al., 2009)(SI, Section 8). D. Still image from a time-lapse of Xenopus CE. Insets indicate representative shortening and nonshortening junctions shown in Panels $\mathbf{E}$ and $\mathbf{F}$ (vertices indicated by arrowheads). $\mathbf{G}$.

Normalized change in length, $L_{n}$, for shortening junctions in vivo (black lines) and in simulations using asymmetric viscoelastic parameters (gray lines) resembling the compressed exponential form (red, dashed line) after the time axis is rescaled. H. Normalized change in length, $L_{n}$, for non-shortening junctions in vivo (black lines) and in simulations using symmetrical viscoelastic parameters (gray lines). I. Quantification of relaxation behavior deviation from the compressed exponential using the residue (SI, Section 9-12). 
A Transverse fluctuations as
proxy for local mechanics

Transverse

movement

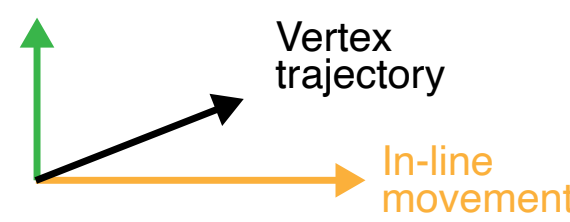

B

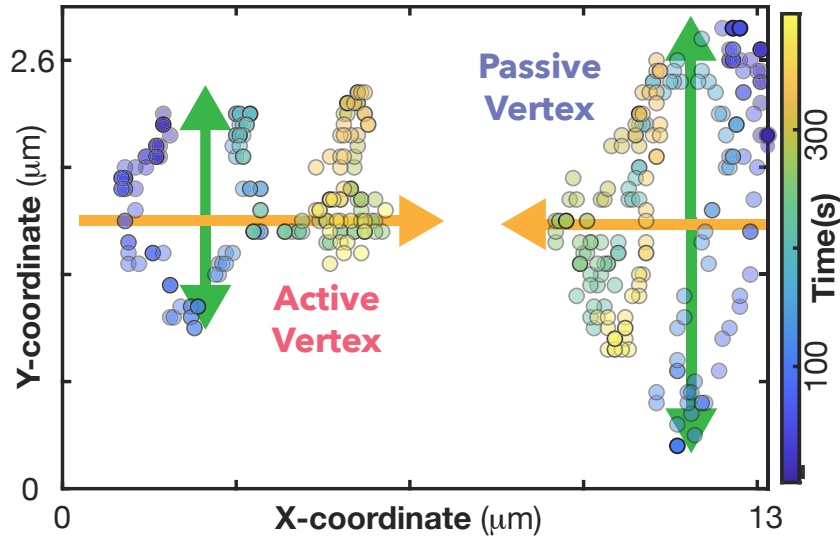

C Mean transverse fluctuations

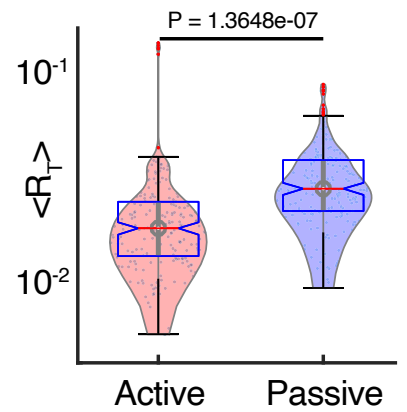

D Probability transverse fluctuations

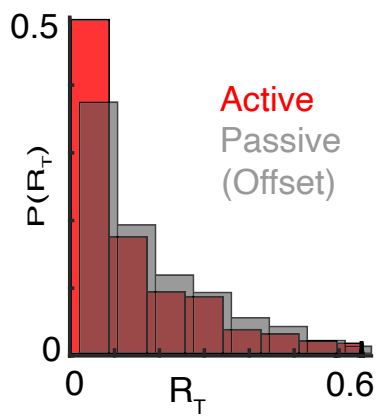

E Mean straightness index

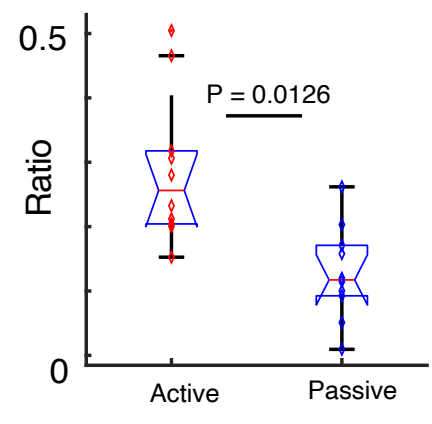

F Probability straightness index

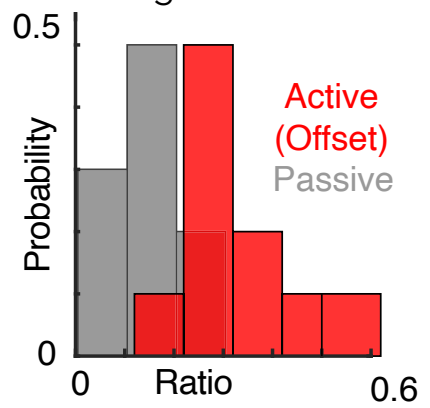


Figure 3. Patterns of transverse vertex fluctuations reveal mechanical heterogeneity of active and passive vertices in vivo.

A. Schematic of transverse fluctuations in the vertex position perpendicular to the direction of junction shortening; traverse movements are extracted using the transverse "hop" function, which is inversely proportional to the local vertex stiffness (SI, Section 14).

B. $X / Y$

coordinates for a representative pair of active and passive vertices color coded for time, with transverse (green) and in-line (orange) motion indicated. C. Mean transverse fluctuation $\left\langle R_{T}\right\rangle$, for active and passive vertices. ( $\mathrm{N}=20$ vertices; 10 embryos over 386 seconds; t-test $P$ value shown). D. Probability distribution of transverse fluctuations, $R_{T}$, (offset for clarity). E. Straightness index quantifying the persistence of vertex motion in terms of directionality (SI, Section 14); t-test $p$ value is shown. F. Probability distribution of the straightness index for active (red, offset for clarity) and passive (blue) vertices. 


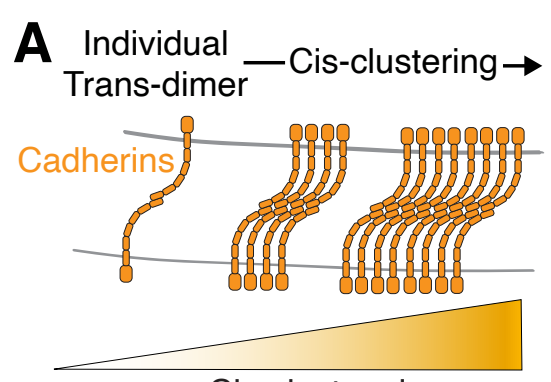

Cis-cluster size

E Single shortening junction

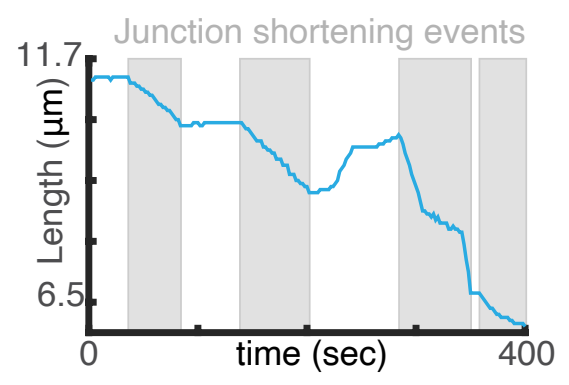

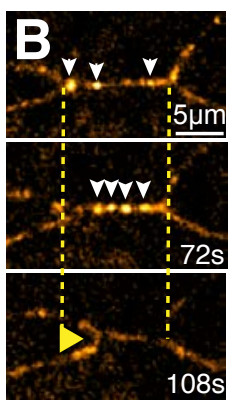

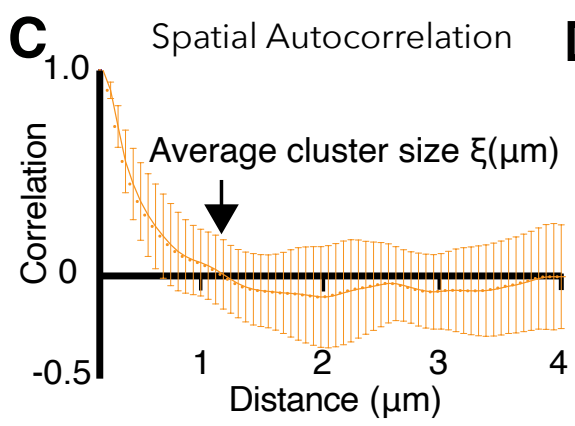

F Cross Correlation Function

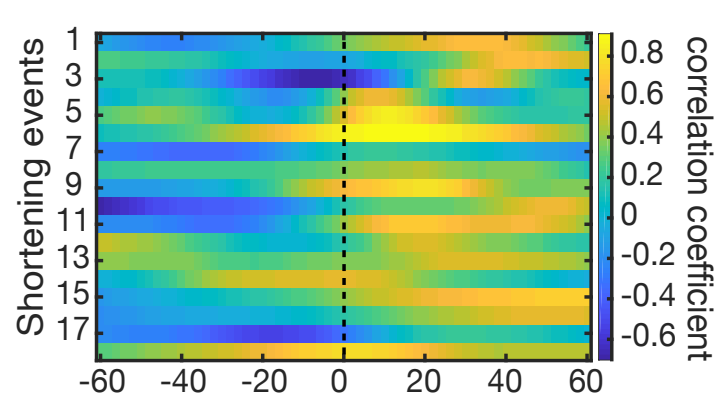

Shortening rate vs cluster size

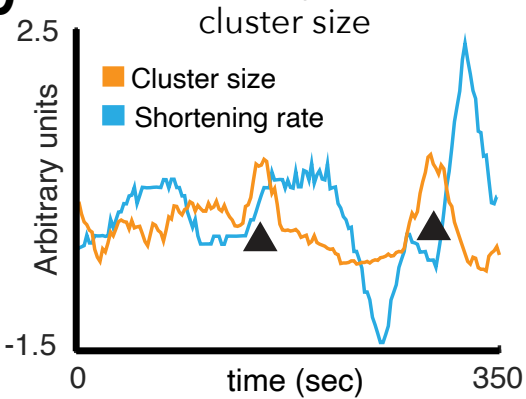

G Cadherin Cluster Size

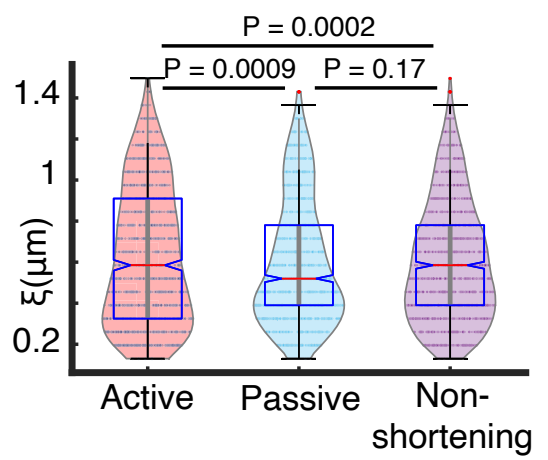


Figure 4. Cadherin cis-clustering correlates with vertex movements and mirrors asymmetric vertex dynamics.

A. C-cadherin (Cdh3) cis-clustering; trans-dimers form across opposing cell membranes (gray); lateral cis interactions drive clustering. B. Frames from time-lapse of Cdh3-GFP; white arrows highlight clusters. Dashed lines denote initial vertex positions; yellow arrow indicates junction shortening. C. Spatial autocorrelation of Cdh3 intensity fluctuations (SI Section 15)(60 image frames, 10 embryos). Autocorrelation decays to zero at $\sim 1 \mu \mathrm{m}$. Error bars are standard deviation. D. Junction length and Cdh3 cluster size fluctuations for an individual cell-cell junction. Cadherin cluster size fluctuations peak prior to junction shortening events. $\mathbf{E}$. Trace from a single $v$-junction displaying pulsatile shortening highlighted by gray boxes. F. Heat map showing cross correlation between junction length and Cdh3 cluster size. Color represents the value of the correlation coefficient (legend at right). Dashed black line indicates zero lag time. (SI, Section 16)( $n=11$ junctions and 18 shortening events.) G. Cadherin cluster size as extracted from spatial correlation curves (Supp. Fig. 6; SI, Section 17). Cadherin cluster sizes are significantly larger near active vertices. Clusters near vertices of non-shortening junctions are not significantly different from those near passive vertices 


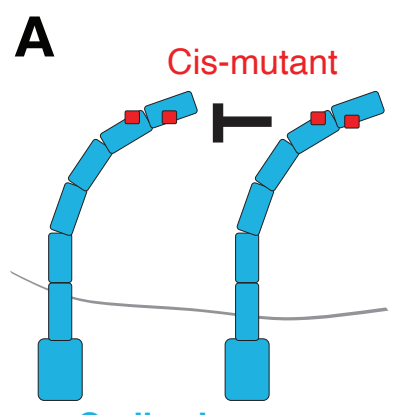

Cadherins
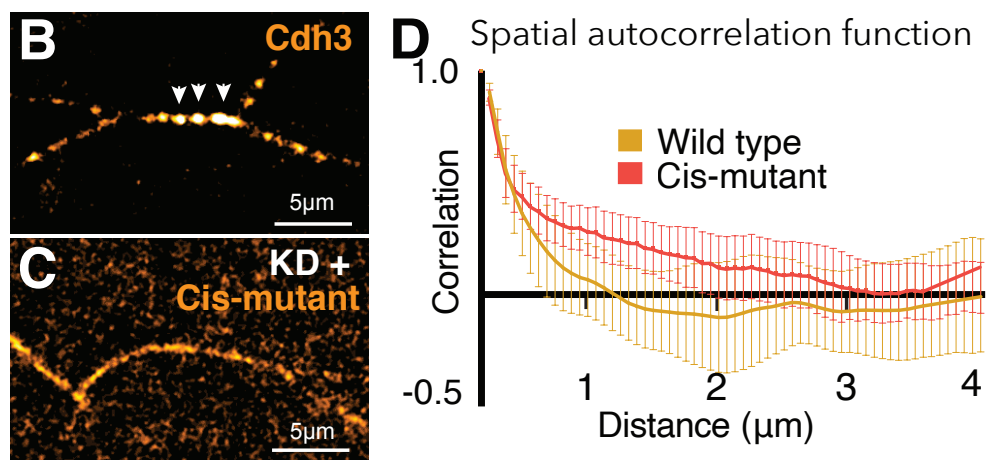
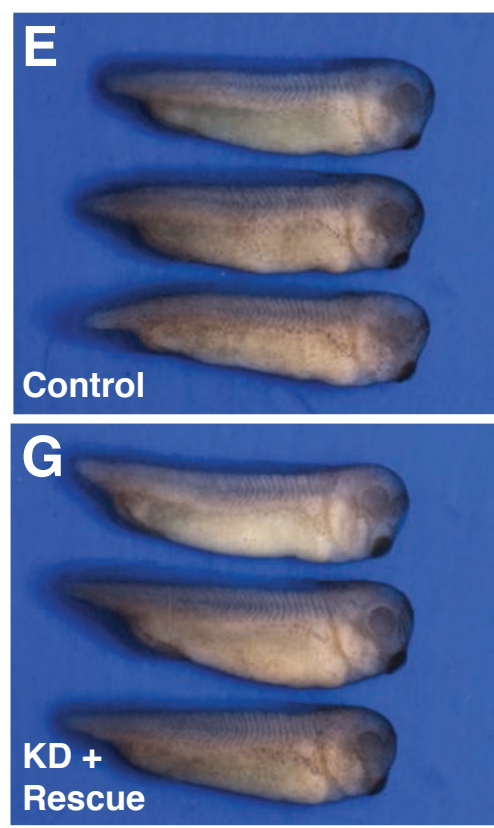

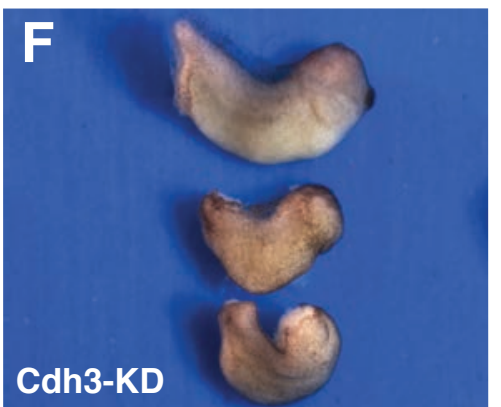

H

KD +

Cis-mutant

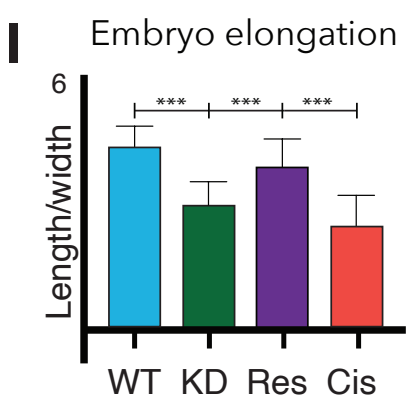

J Embryo cohesion

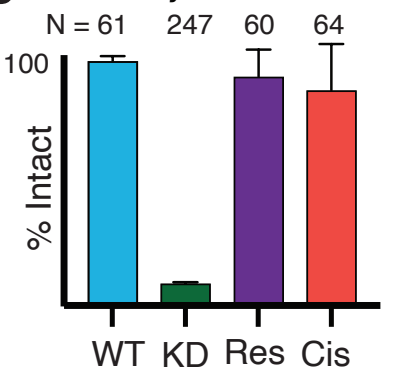


Figure 5. Cdh3 cis-clustering is required for convergent extension but not homeostatic tissue cohesion.

A. Mutations used to inhibit cadherin cis-clustering. B. Cdh3-GFP clustering in a control embryo. C. Cis-clusters absent after re-expression of cisMut-Cdh3-GFP. D. Mean spatial autocorrelation of Cdh3-GFP intensity fluctuations for wild type (60 image frames, from 10 embryos) and the cis-mutant (56 image frames, 5 embryos) (SI, Section 18). Gradual, nonexponential decay for cisMut-Cdh3-GFP indicates a lack of spatial order (i.e. failure to cluster). E. Control embryos ( stage 33). F. Sibling embryos after Cdh3 knockdown. G. Knockdown embryos re-expressing wild-type Cdh3-GFP. H. Knockdown embryos re-expressing cisMutCdh3-GFP. I. Axis elongation assessed as the ratio of anteroposterior to dorsoventral length at the widest point. J. Embryo cohesion assessed as percent of embryos alive and intact at stage 23. 

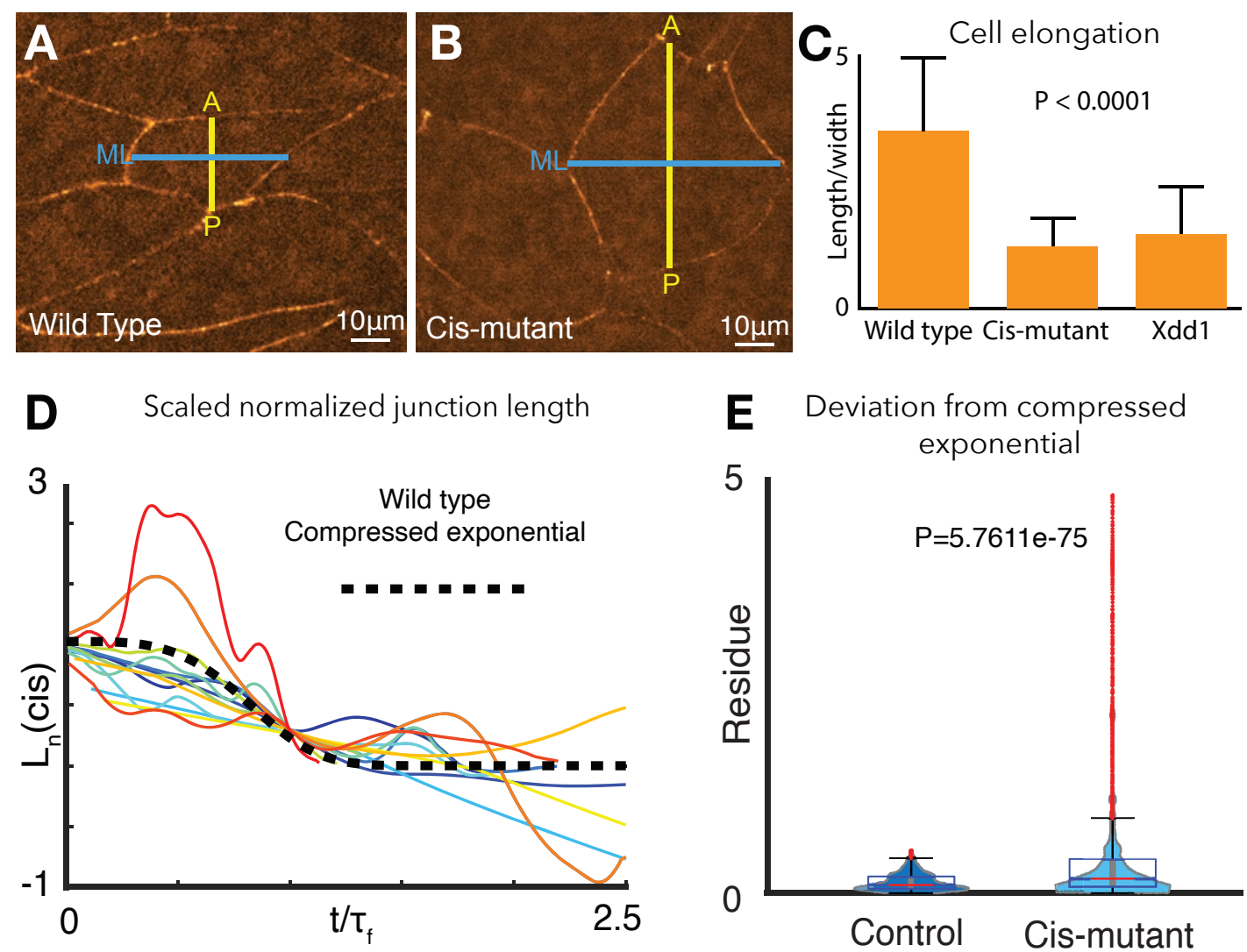

F Mean Transverse Fluctuations
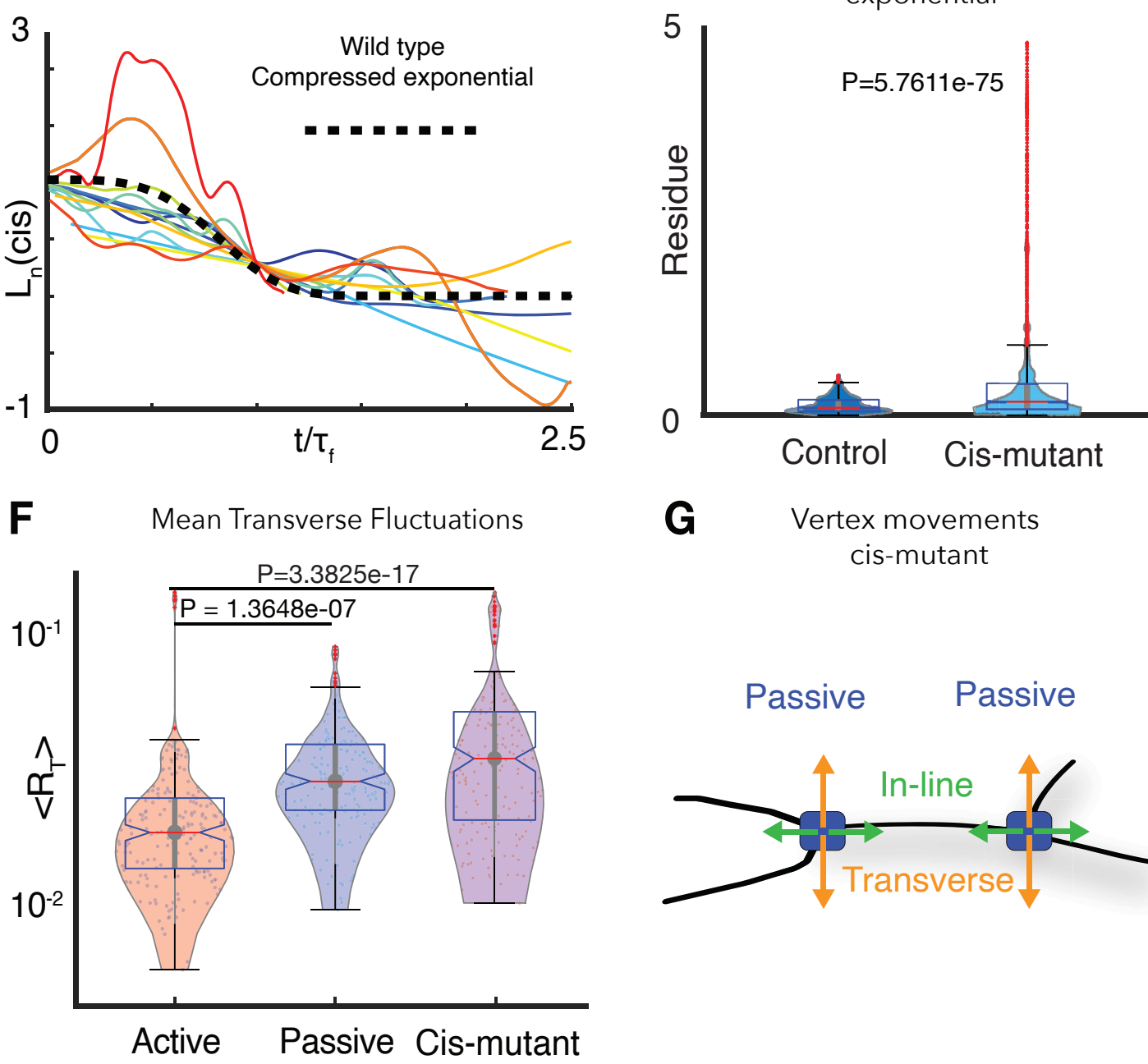

G Vertex movements cis-mutant

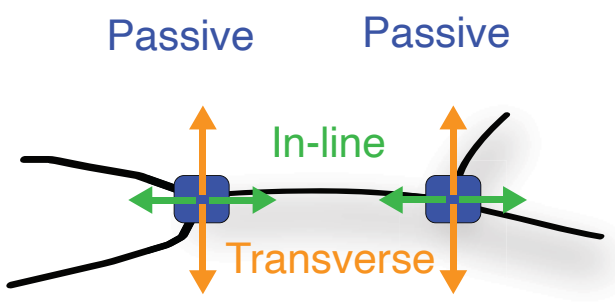


Figure 6. Cdh3 cis-clustering is required for heterogeneous junction mechanics.

A. Image of polarized, elongated control Xenopus mesoderm cells. Blue = mediolateral $(\mathrm{ML})$; yellow = anterior-posterior (AP). B. Stage-matched cells after depletion of endogenous Cdh3 and re-expression of cisMut-Cdh3. C. Cellular length/width ratio to quantify CE cell behaviors ( $p$ value indicates ANOVA result). D. Normalized junction length dynamics $\left(L_{n}\right)$ for cis-mutant expressing junctions. Large fluctuations here are similar to those seen normally in nonshortening junctions (see Fig. $2 \mathrm{H}$ ). Dashed black line indicates the expected compressed exponential. E. The residue quantifying significant $L_{n}$ deviation from the compressed exponential function as compared to control junctions. F. Plots for transverse fluctuations, $\left\langle R_{T}\right\rangle$, for control active and passive vertices compared to cis-mutant vertices. (Note: Data for active and passive junctions are re-presented from Fig. 3C for comparison.) G . Schematic illustrating symmetrical vertex behavior after disruption of cdh3 cis-clustering. 
A Planar cell polarity
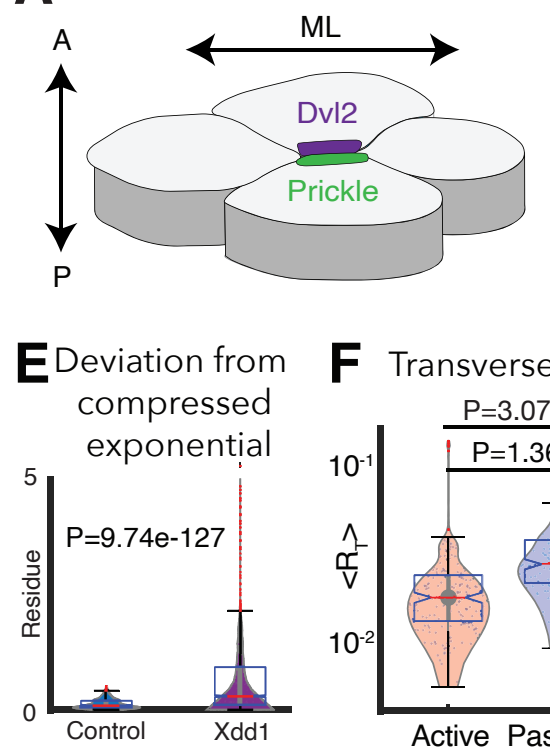
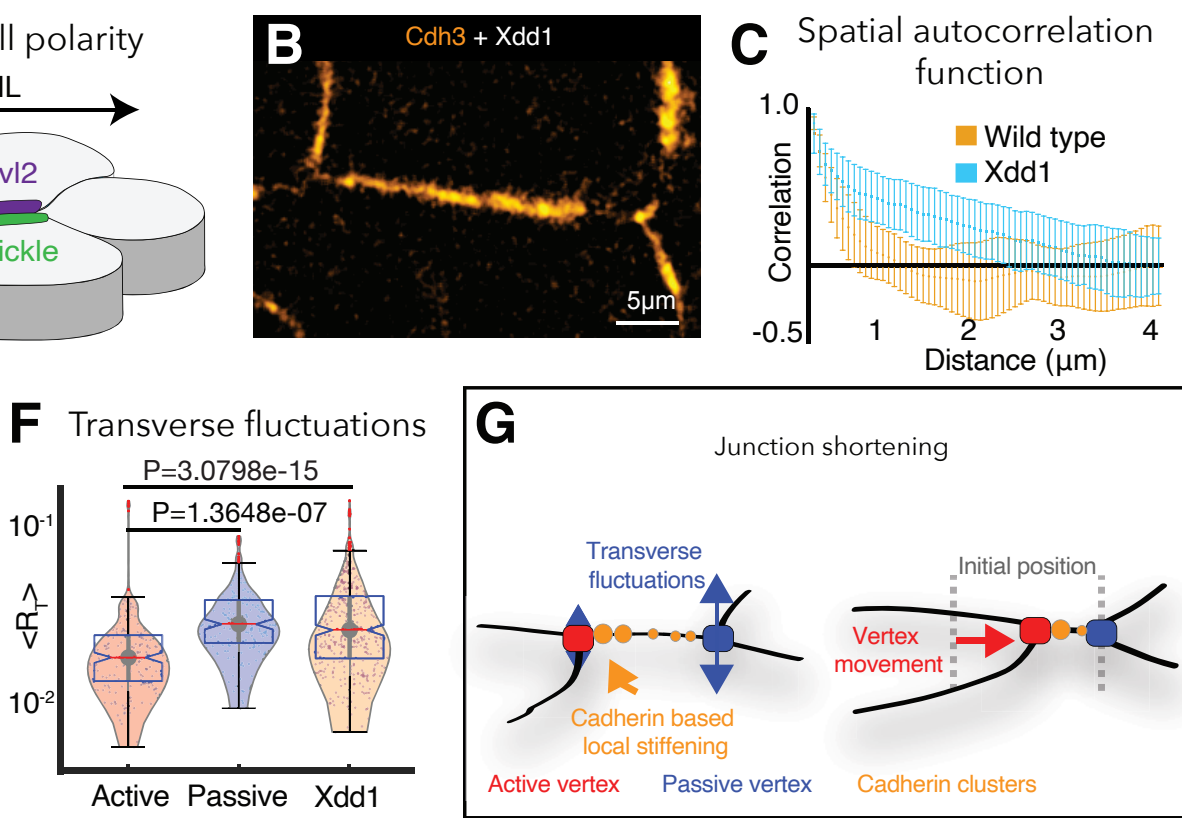
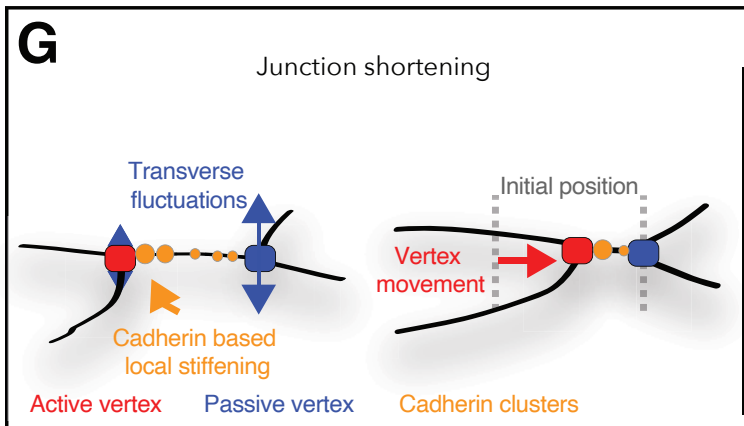

D Scaled normalized junction length

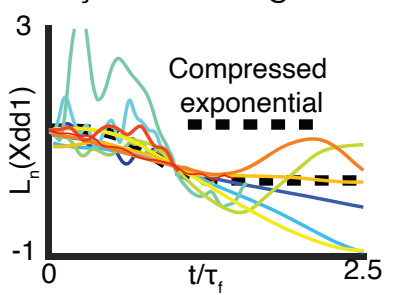

Non-shortening junctions Cis-mutant or Xdd1

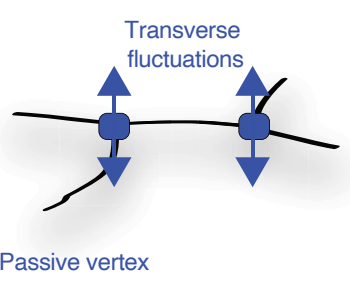


Figure 7. PCP is required for cdh3 cis-clustering and heterogeneous junction mechanics.

A. Cartoon of polarized core PCP protein localization. B. Still image of Cdh3-GFP after expression of dominant negative Dvl2 (Xdd1). C. Spatial autocorrelation of Cdh3 intensity fluctuations for Xdd1 (53 image frames, 5 embryos) and control embryos (60 frames, from 10 embryos), +/- std. dev. The spatial organization of Xdd1 mutant cadherin is similar to cisMutCdh3 expressing embryos. D. Normalized junction length dynamics for Xdd1 embryos. Dashed black line indicates the normal compressed exponential behavior. E. Residue for the deviation from the universal compressed exponential function for Xdd1 junctions. F. Plots for transverse fluctuations at active and passive vertices compared to Xdd1-expressing vertices. (Note: Data for active and passive junctions are re-presented from Fig. $3 \mathrm{C}$ for comparison to Xdd1.) $\mathbf{G}$. Schematic summarizing the primary conclusions. 


\section{Supplemental figure 1}

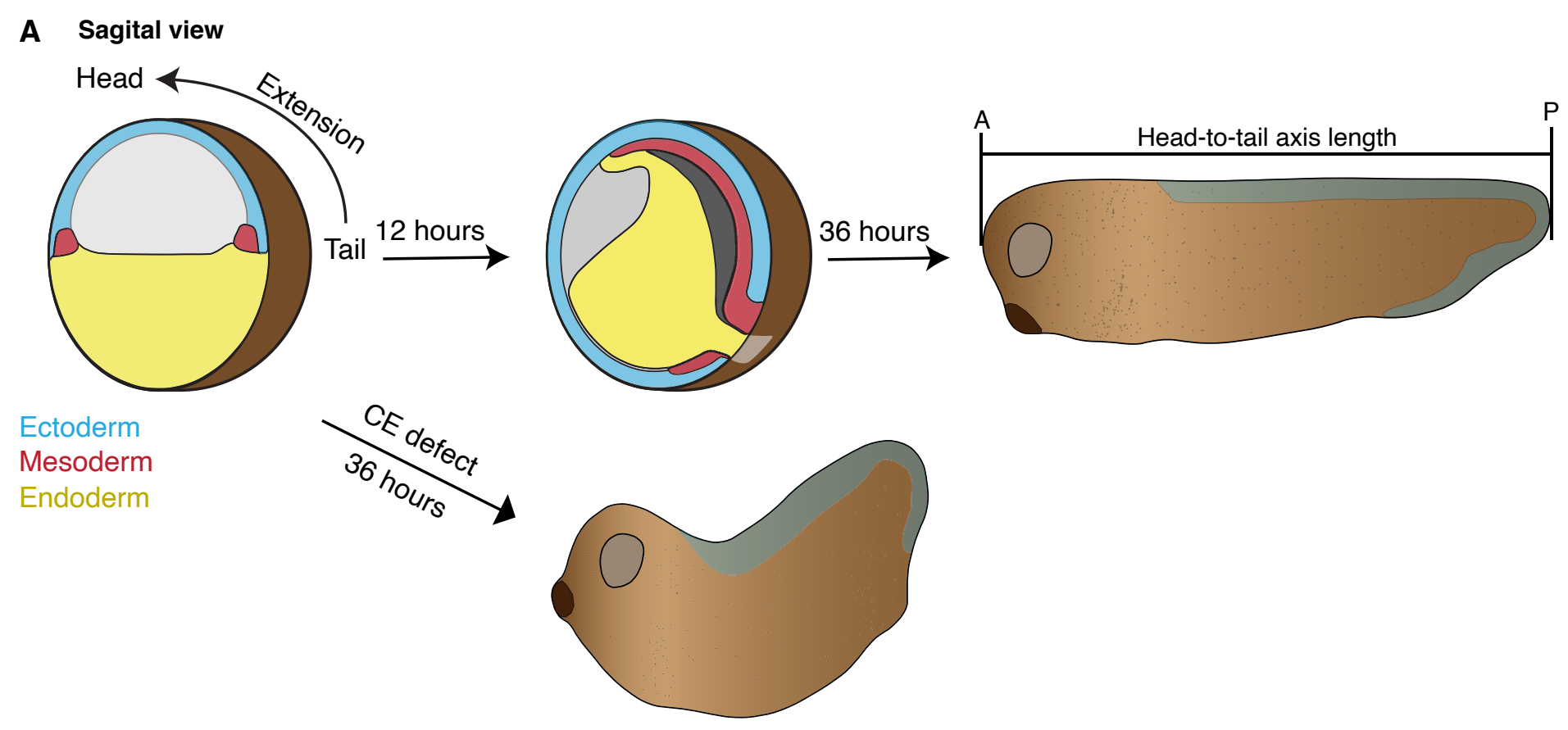

\section{B}

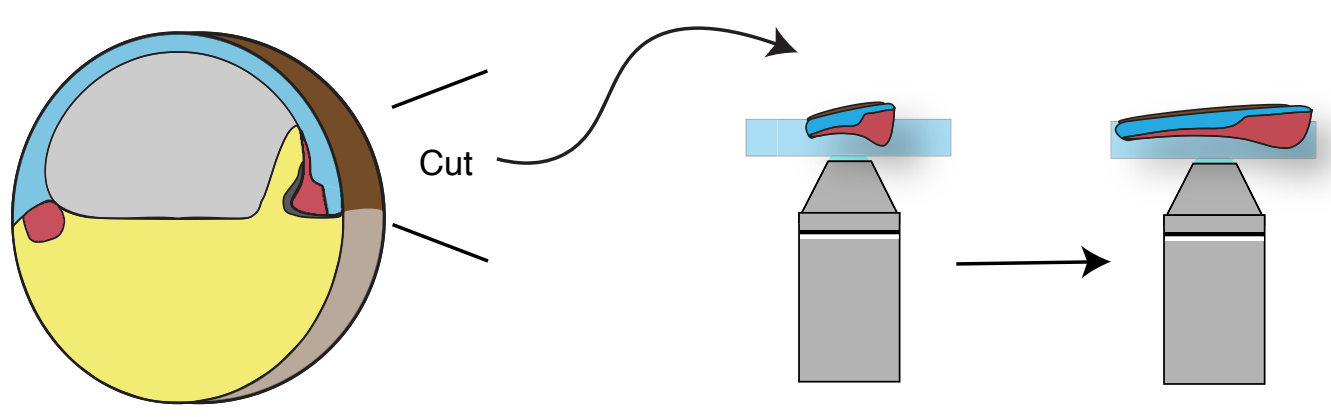

\section{Ectoderm}

Mesoderm

Endoderm 


\section{Supplemental Figure 1: Schematics of Xenopus development.}

A. Cartoon depiction of Xenopus gastrulation, tadpole axis elongation, and the consequence of convergent extension defects. Here the mesoderm in the dorsal marginal zone (DMZ) involutes and undergoes convergent extension to establish the animals anterior-posterior (head-to-tail) axis. This axis will then continue to elongate during tadpole stages. Disruption of CE results in stunted embryos with a classic 'swayed back' appearance (lower arrow). B. Embryological techniques were used to excise the DMZ (Keller explant) allowing visualization of CE in realtime. 


\section{Supplemental figure 2}

A

Mean Square Displacement (MSD)

of Individual verticies

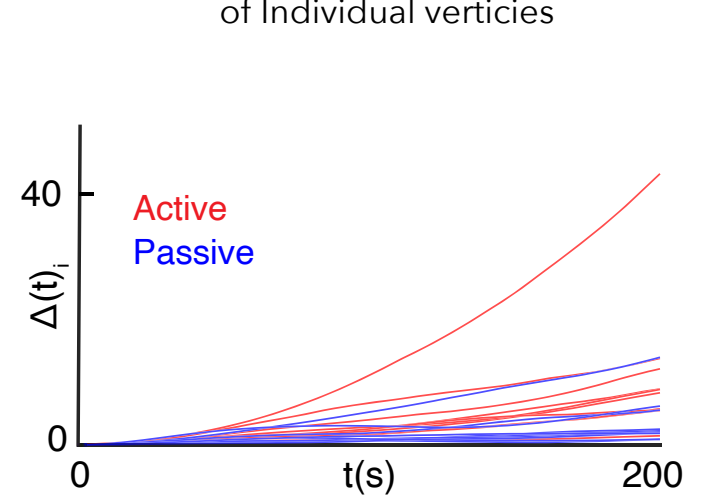

B MSD Lab frame of reference

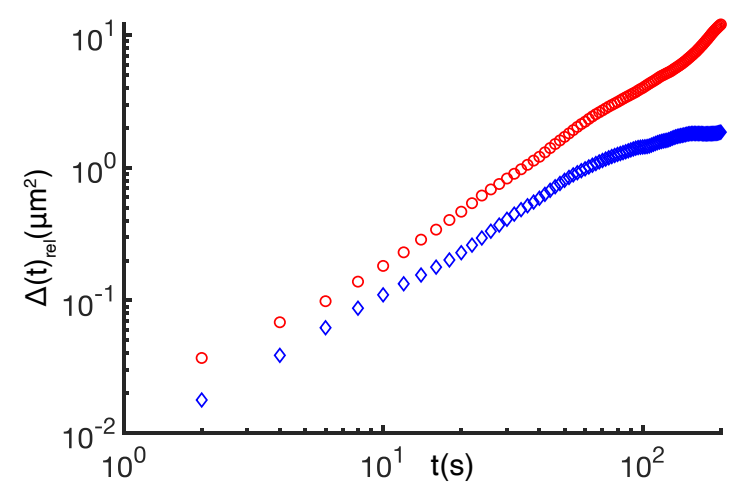

C Cell Frame D Relative MSD in the E of Reference Cell Frame of Reference
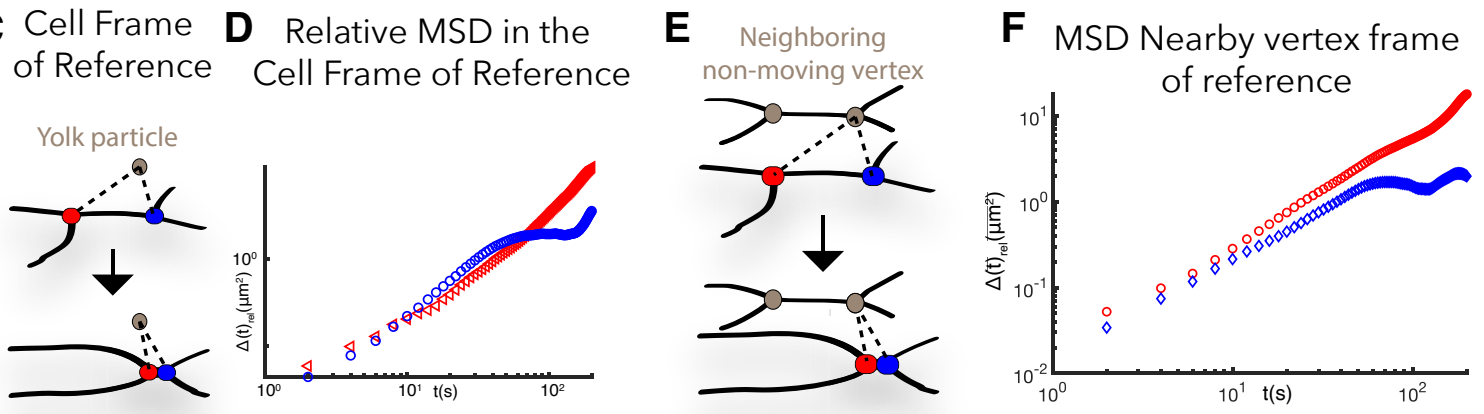


\section{Supplemental Figure 2: Mean squared displacement measured from multiple frames of reference.}

A. Mean squared displacements (MSD), $\Delta(t)_{i}$, of 10 distinct individual active (red) and passive (blue) vertices. Heterogeneity in individual vertex movements are apparent from the wide variation in individual vertex MSDs. See Theory SI Section 1 for details. B. Mean squared displacement of a representative pair of active and passive vertices in the "lab" frame of reference (i.e. as they appear in raw images). C. Schematic showing how vertex mean square displacement was measured relative to yolk particles that are present in the embryonic Xenopus cells. We refer to this as the relative MSD in the "cell" frame of reference, which eliminates the effect of translational and rotational motion of the tissue during time-lapse imaging. D. Relative mean squared displacement of representative vertices in the cell frame of reference. $\mathbf{E}$. Schematic showing how vertex mean square displacement was measured relative to a neighboring vertex from other cells ("nearby vertex" frame of reference). F. Relative mean squared displacement of the same pair of active and passive vertices in $D$ (above) in the nearby vertex frame of reference. 


\section{Supplemental figure 3}
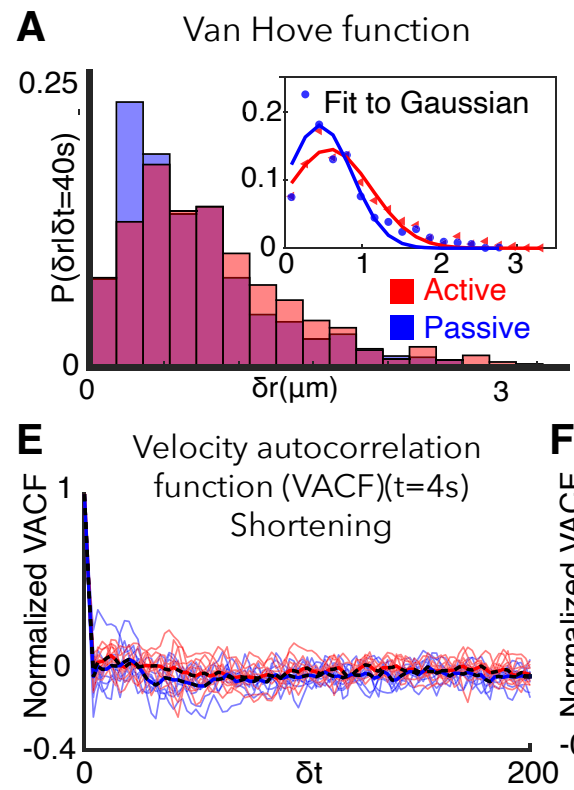

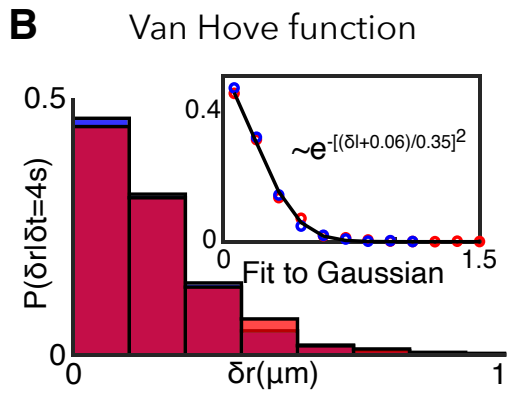

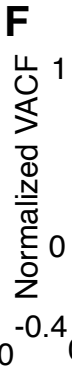

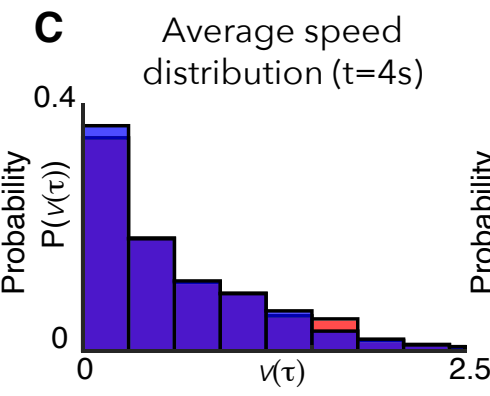

G Self-Overlap Parameter

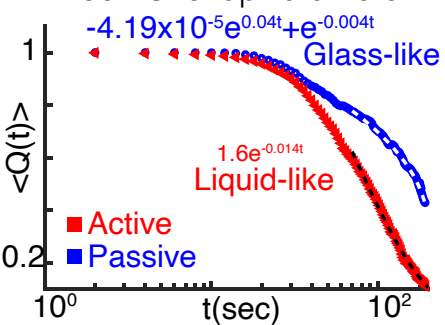

D Average speed distribution ( $\mathrm{t}=60 \mathrm{~s})$

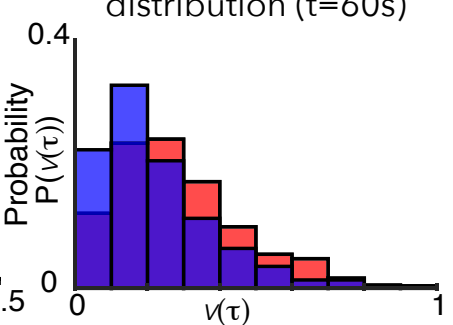

H Self-Overlap Parameter

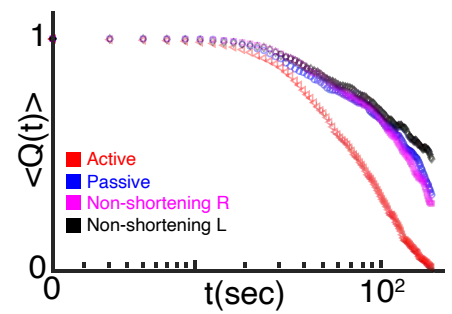

I Dynamic Heterogeneity
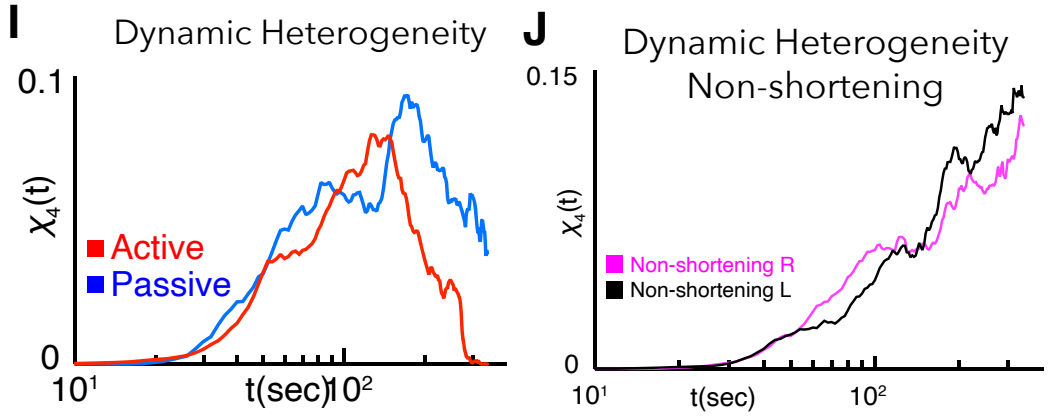


\section{Supplemental Figure 3: Extended analysis of vertex glass-like dynamics.}

A. Probability distribution of active (red) and passive (blue) vertex displacements, referred to as the van Hove function (SI Section 2), shows distinct non-Gaussian functional form. Inset shows deviation of the van Hove function from fits to Gaussian function $P(\delta r)=0.14 e^{-\left(\frac{\delta r-0.58}{0.76}\right)^{2}}$ for the active vertex and $P(\delta r)=0.18 e^{-\left(\frac{\delta r-0.46}{0.59}\right)^{2}}$ for the passive vertex. Probability distribution of distances moved over a time scale of $\delta t=40 \mathrm{~s}$, clearly shows the enhanced distance moved by active vertices as compared to passive vertices. Probability distribution is obtained for 20 individual vertices from 10 embryos. B. Van Hove distribution of active (red) and passive (blue) vertex displacements at $\delta t=4 s$. Van Hove distribution for both vertices are well-fit by a Gaussian distribution, indicating normal diffusive movement at short time scales. C. Average vertex speed distribution for active (red) and passive (blue) vertices at $\tau=4 \mathrm{~s}$ (SI, Section 2). The speed distribution peaks at low values of average speed and rapidly decays of zero, showing similar trends for both active and passive vertices at $\tau=4 s$. D. Average vertex speed distribution vertices at $\tau=60 \mathrm{~s}$. The distribution peaks at intermediate values. The active vertex speed distribution decays slower for larger values of the average velocity compared to passive vertices, indicating enhanced movement of active vertices. E. Velocity autocorrelation function (VACF) for active (red) and passive (blue) vertices at $\tau=4 s$ (SI, Section 42). Active and passive vertex velocity autocorrelation rapidly decay to zero over a time scale of $\sim 5 s$. Individual vertex correlations are plotted as solid lines. Mean is plotted as dashed lines. Active and vertex velocity correlations are similar in time at a short time interval, $\tau=4 \mathrm{~s}$. F. Velocity autocorrelation function (VACF) for active (red) and passive (blue) vertices at $\tau=60 \mathrm{~s}$ (see SI, Section 4). Active vertex velocity is more persistent in time at this longer time interval compared to the passive vertex velocity. VACF quantifies the emergence of persistent motion of the active vertex at $\tau=60 \mathrm{~s}$. The persistence time of the velocity correlation is over $50 \mathrm{~s}$. $\mathbf{G}$. The overlap of vertex positions, as quantified by the self-overlap parameter (SI, Section 3$)\langle Q(\delta t)\rangle$ decays rapidly to zero for active vertices (red). The tail of the self-overlap parameter is well fit by an exponential function for active vertices, indicative of fluidized dynamics. For passive vertices, however, the decay of the self-overlap order parameter is better fit to a double exponential, indicative of glass-like slowdown in the dynamics. 20 individual vertices from 10 embryos are analyzed. $\mathbf{H}$. For non-shortening vertices (magenta, black), the overlap with its initial positions, as quantified by $\langle Q(\delta t)\rangle$ decays slower compared to active vertices (red). The overlap decays to 0 in the time interval probed for active vertices. The decay in the self-overlap parameter for passive vertices are comparable to non-shortening left and right vertices. I. The four-point susceptibility (SI Section 4), $\chi_{4}(\delta t)=\left\langle Q(\delta t)^{2}\right\rangle-\langle Q(\delta t)\rangle^{2}$, is calculated from the moments of $\langle Q(\delta t)\rangle$. The time at which $\chi_{4}(\delta t)$ peaks correspond to the characteristic lifetime of correlated motion of vertices contributing to CE. Red curve is for the active vertex and blue for passive vertex. 20 individual vertices from 10 embryos are analyzed. $\mathbf{J}$. The four-point susceptibility, $\chi_{4}(\delta t)=\left\langle Q(\delta t)^{2}\right\rangle-$ $\langle Q(\delta t)\rangle^{2}$, is calculated from the moments of $\langle Q(\delta t)\rangle$. The $\chi_{4}(\delta t)$ for non-shortening left (black) and right (magenta) vertices do not show a peak in the time frame analyzed. 


\section{Supplemental figure 4}

A Normalized Junction Length

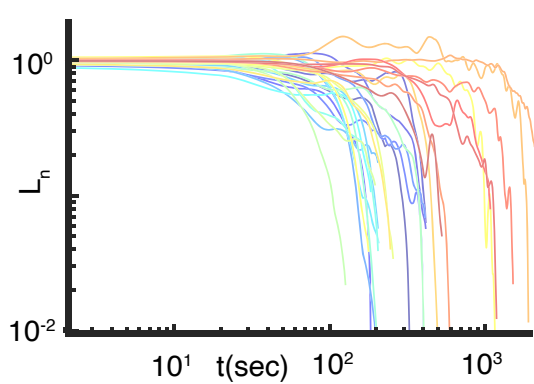

C Scaled normalized Junction Length

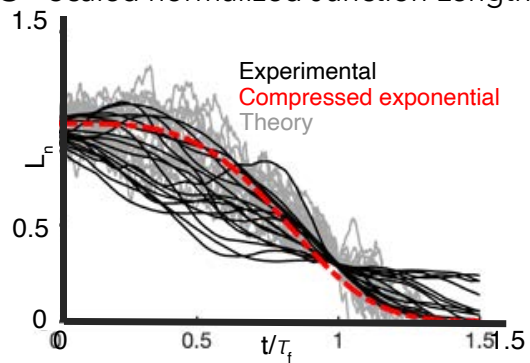

B Rescaled Normalized Junction Length

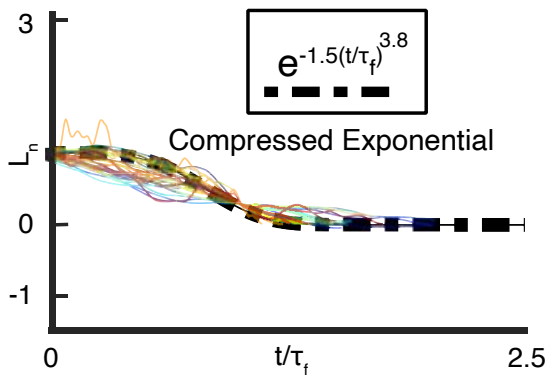

D Phase Space of Theory Parameters

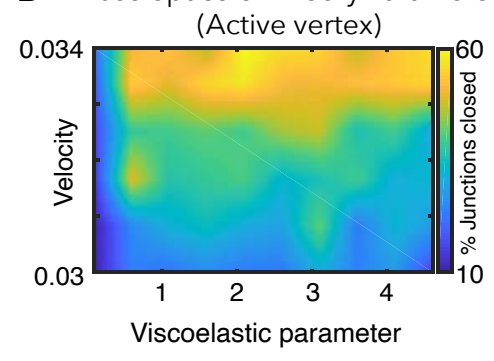




\section{Supplemental Figure 4: Extended analysis comparing in vivo and in silico junction dynamics}

A. Normalized relative change in length, $L_{n}(t)=\frac{L(t)-L\left(t_{f}\right)}{L\left(t_{o}\right)-L\left(t_{f}\right)}$, versus time for shortening mediolateral cell-cell junctions during CE. 21 individual junctions from 20 embryos are analyzed. $L\left(t_{0}\right)$ and $L\left(t_{f}\right)$ are the junction lengths at initial time $t_{0}$ and final time $t_{f}$ respectively. B. Although the normalized lengths vary considerably, the $L_{n}$ nearly collapse onto a single universal curve (black dashed line) when the time axis is scaled by the relaxation time $\tau_{f}$. The relaxation time is defined as $L_{n}\left(t=\tau_{f}\right)=0.3$, suggesting an underlying self-similarity of the cell rearrangement process contributing to $\mathrm{CE}$. C. Comparison between experimental (black) and theoretical (grey) normalized junction length vs time for shortening junction shows that the model captures experimentally observed features of junction shortening during convergent extension. D. Phase diagram for an alternative model (SI, Section 13) of active versus passive vertex contribution to junction shortening. Instead of accelerating vertices, we consider the velocity of vertices to be constant. Active vertex velocity is taken to be larger than the passive vertex. Simulations using the alternative model tell us that the viscoelastic parameter controls junction shortening. Our conclusion on the importance of the viscoelastic parameter in effecting junction shrinking is independent of the details of the vertex dynamics. 


\section{Supplemental figure 5}
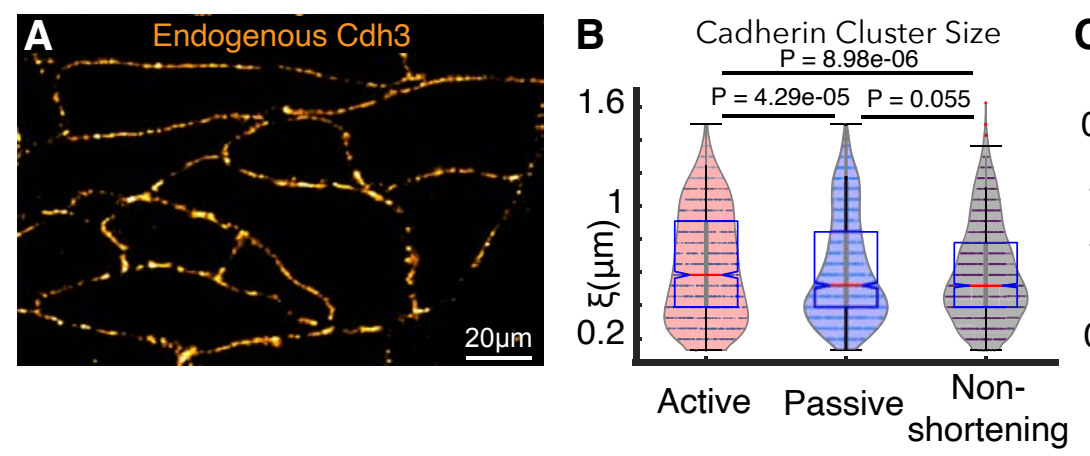

C Cadherin Cluster Size

D Cadherin Cluster Size
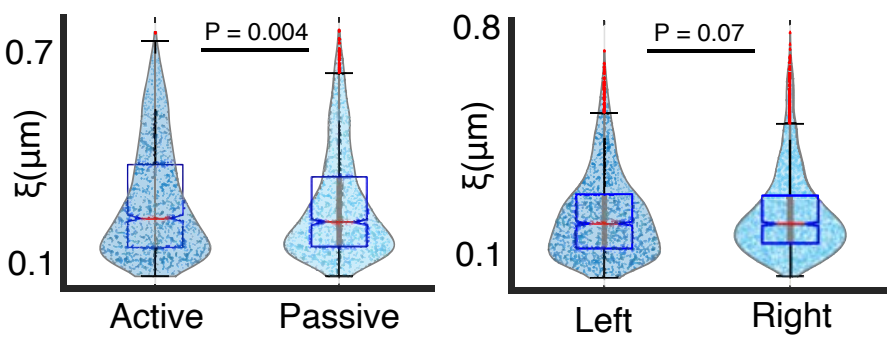


\section{Supplemental Figure 5: Extended analysis pertaining to Cdh3 clustering}

A. Immunostaining for endogenous $\mathrm{Cdh} 3$ showing that the endogenous protein forms cadherin cis-clusters. B. Violin plot of Cdh3 cluster sizes next to active vertices (red), passive vertices (blue), and non-shortening left and right vertices (black) for all frames including shortening and non-shortening events. Statistical significance was assessed using a Kolmogorov-Smirnov test. C. An alternative definition of cluster size was used to analyze differences in cadherin clustering (SI, Section 17). By fitting an exponential function to the spatial autocorrelation of cadherin intensity fluctuation, we extract the characteristic cadherin cluster size. Violin plot shows the Cdh3 cluster sizes for active versus passive vertices in shortening junctions. 1538 frames from 10 embryos were analyzed. Kolmogorov-Smirnov shows significant differences between Cdh3 clustering near active versus passive vertices $(p=0.004)$. D. Violin plot of Cdh3 cluster sizes for left versus right vertices in non-shortening junctions. 2062 frames from 7 embryos were analyzed. Kolmogorov-Smirnov did not indicate significant differences between Cdh3 clustering near left versus right vertices $(p=0.07)$. 


\section{Supplemental figure 6}

bioRxiv preprint doi: https://doi.org/10.1101/2020.02.11.944033; this version posted November 16, 2020. The copyright holder for this preprint (which was not certified by peer review) is the author/funder. All rights reserved. No reuse allowed without permission.
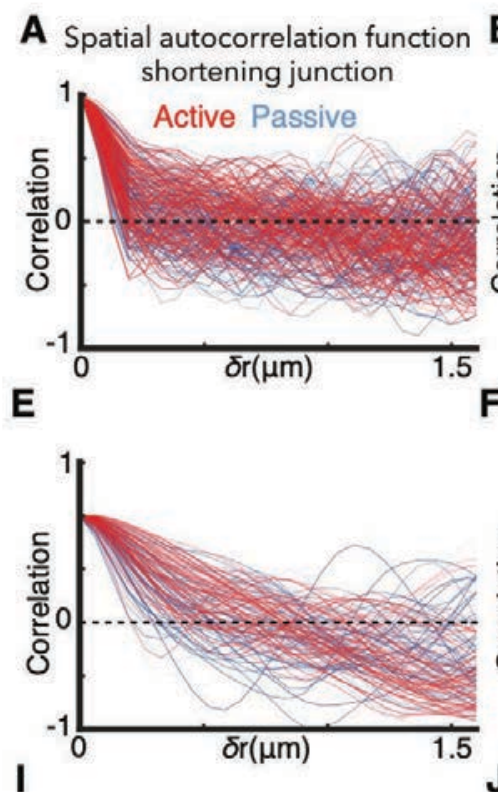

I

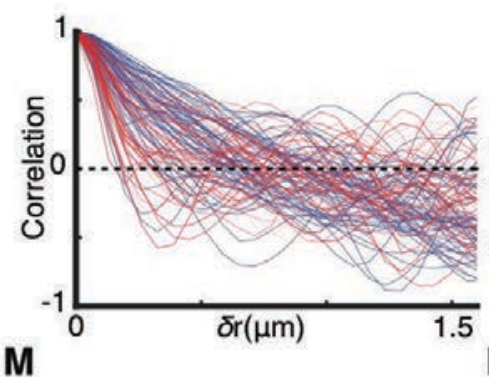

M

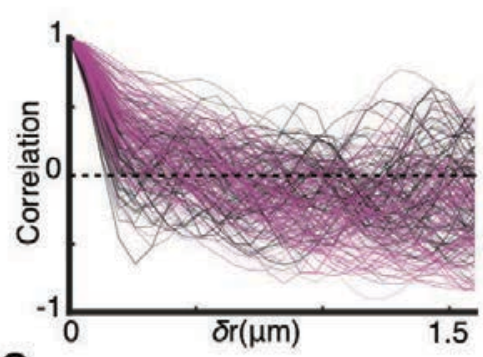

Q

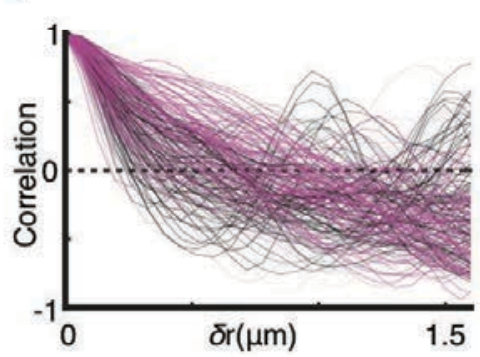

C
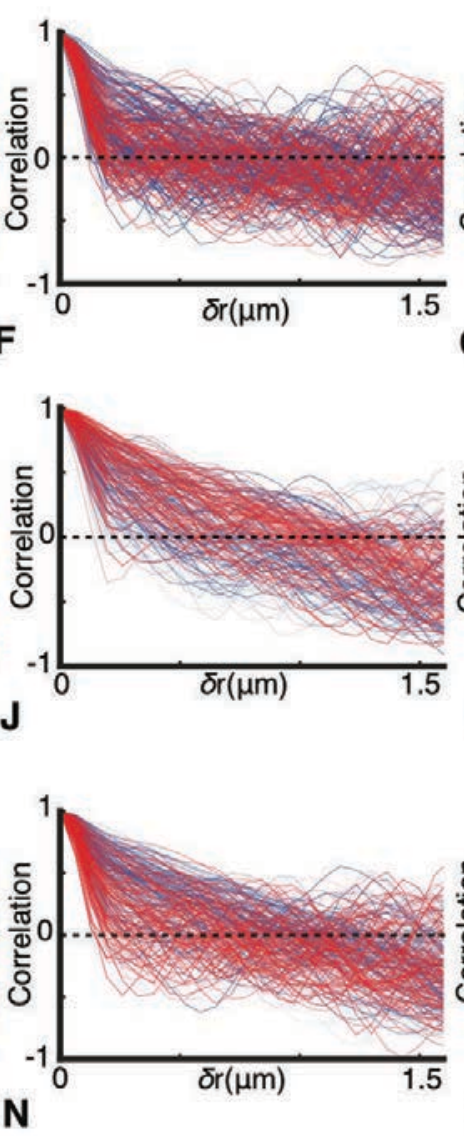

N

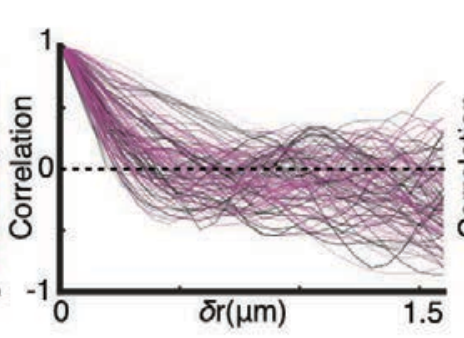

D
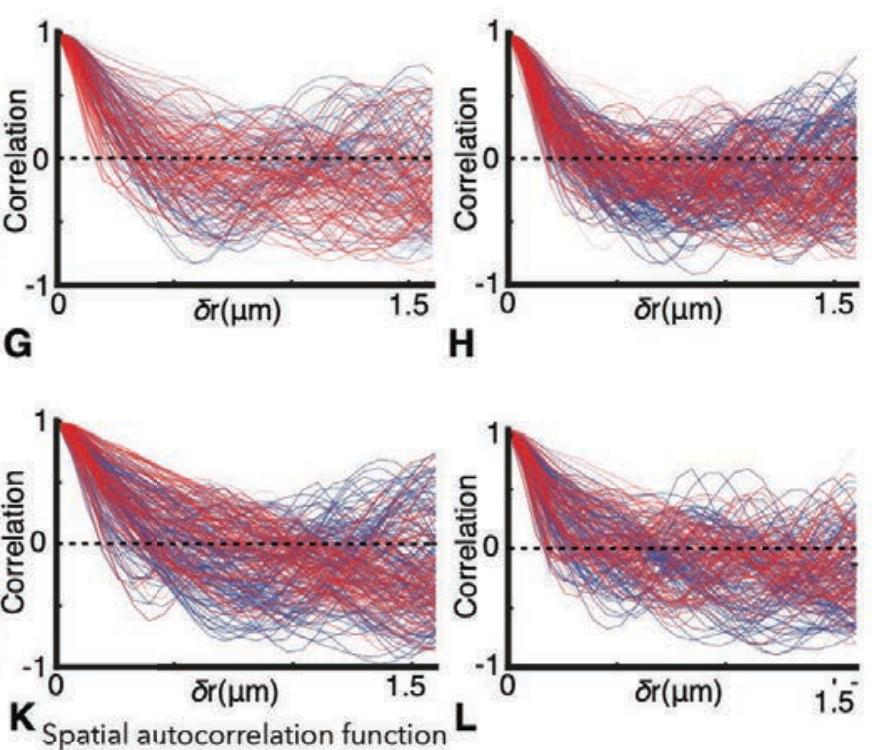
Non-shortening junction
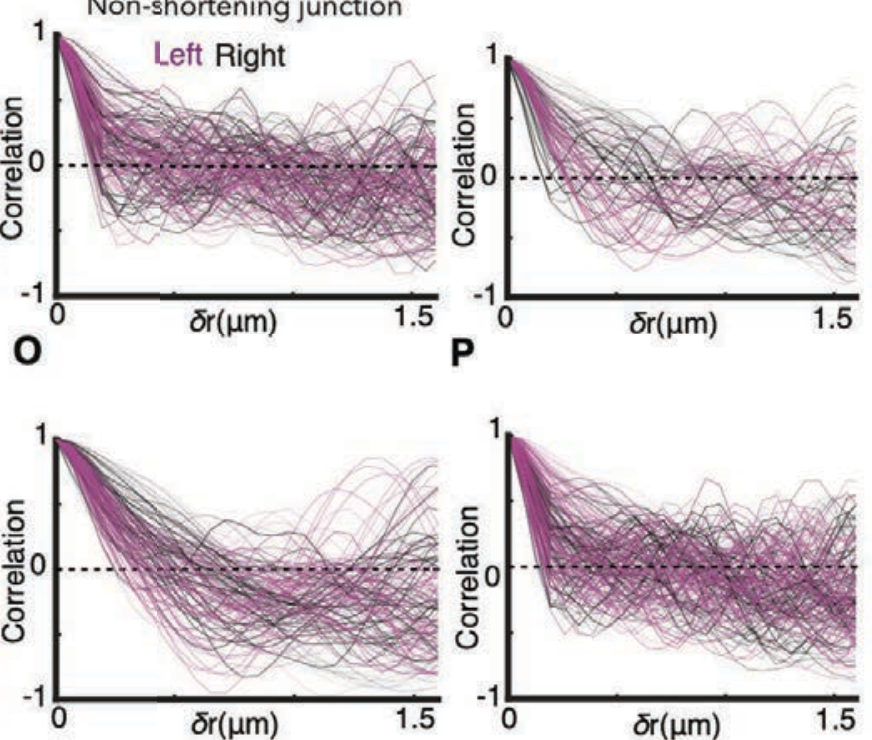
Supplemental Figure 6: Source data for spatial correlation of Cdh3 intensity fluctuations reveal extreme heterogeneity in cluster size.

A-J. Individual (time frame by frame) spatial correlation vs distance curves selecting for shortening events from 10 distinct cell-cell junctions that undergo successful junction shortening (E-N). Cadherin spatial correlation near active (passive) vertices are shown in red (blue) lines. K-Q. Individual (time frame by frame) spatial correlation vs distance curves from 7 distinct cellcell junctions that do not successfully shorten (O-U). Cadherin spatial correlation near left (right) vertices are shown in magenta (black) lines. 


\section{Supplemental figure 7}
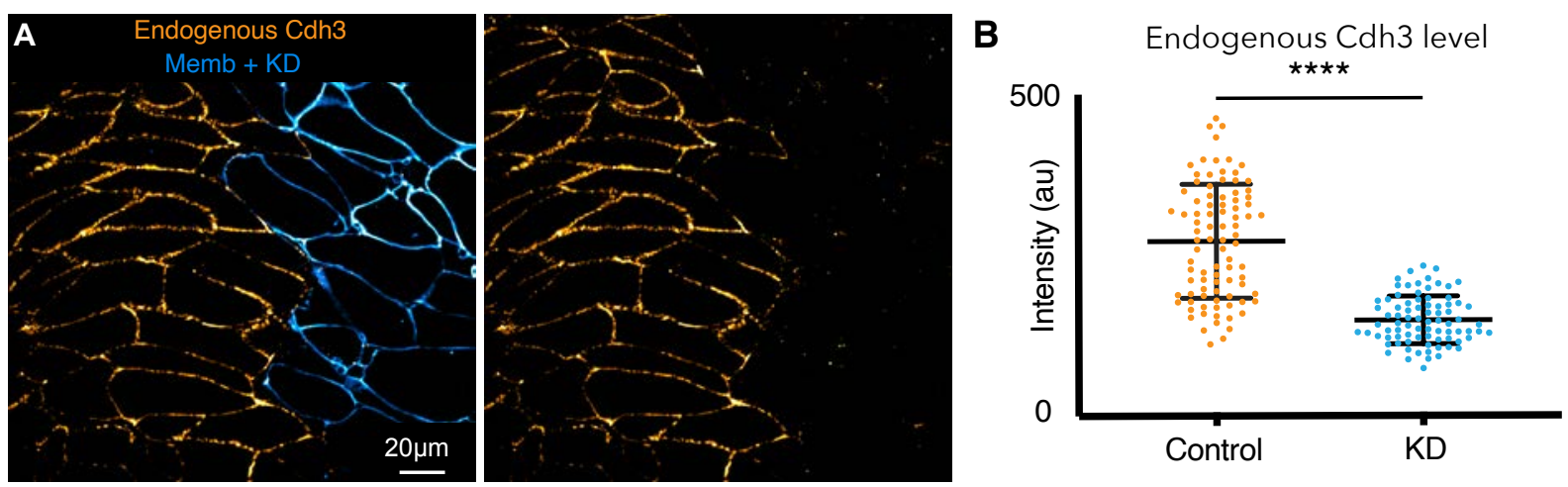


\section{Supplemental Figure 7: Cdh3 knockdown.}

A. Embryos were injected with Cdh3-MO and membrane-BFP in a single dorsal blastomere at the 4-cell stage resulting in mosaic depletion of Cdh3. Here immuno-staining for Cdh3 shows that the protein was depleted specifically in cells that received the morpholino, as marked by membrane-BFP. B. Cdh3 immuno-staining intensity was measured in control cells and neighboring Cdh3-MO cells from mosaic animals. These data were derived from three replicate experiments and statistically analyzed by t-test 


\section{Supplemental figure 8}

A Spatial autocorrelation function B Mean spatial autocorrelation function

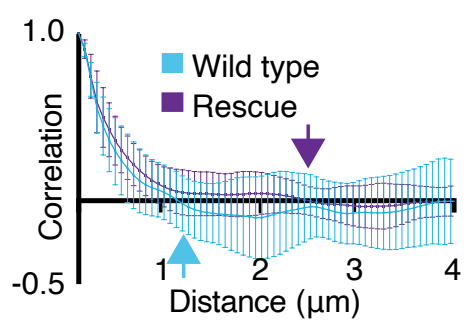

E Spatial autocorrelation function for single junctions

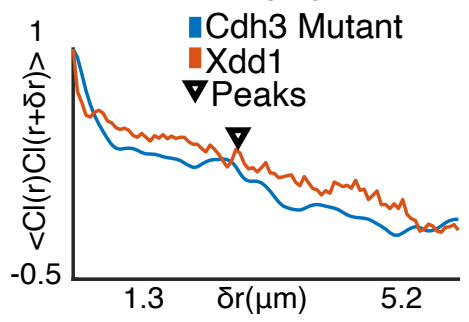

F Spatial autocorrelation
C Spatial autocorrelation D
function for single junctions

Spatial autocorrelation function for multiple junctions
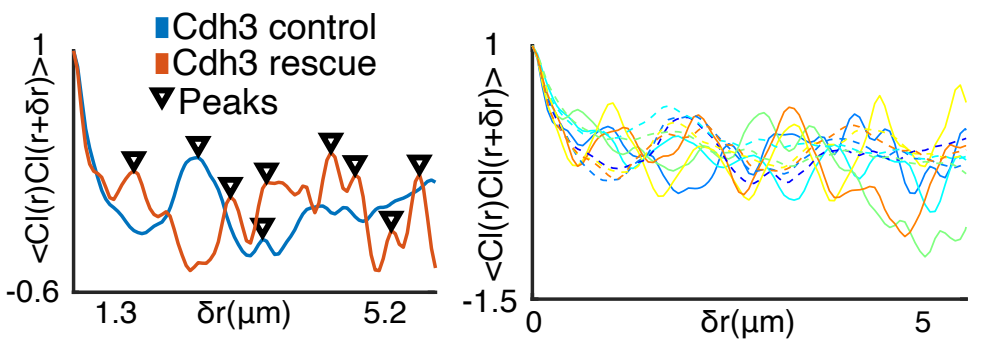
function for multiple junctions

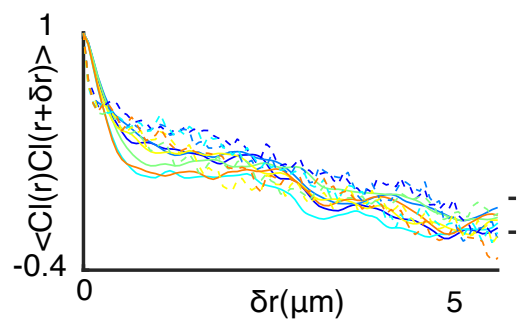

G Mean spatial autocorrelation function

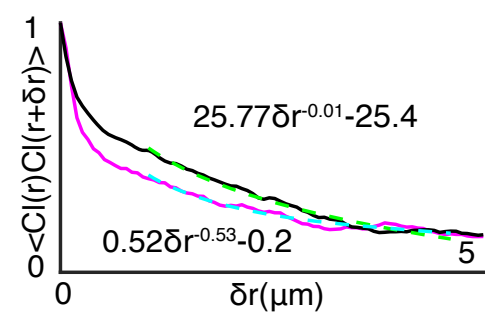




\section{Supplemental Figure 8: Extended analysis of cadherin clustering for the cis-mutant, rescue, and Xdd1.}

A. Spatial autocorrelation of Cdh3 intensity fluctuations for wild type (60 frames, obtained from 10 embryos) and Cdh3-rescue (58 frames, obtained from 4 embryos). The characteristic correlation length decays to zero at $\sim 1 \mu \mathrm{m}$, for both wild type and rescue embryos. Error bar is the standard deviation. B. Mean spatial autocorrelation of Cdh3 intensity fluctuations for wild type and rescue with functional fits to the decay behavior. Both spatial correlations can be fit to an exponential. This is evidence for a characteristic spatial scale for the correlation in spatial Cdh3 intensity fluctuations. C. Single junction spatial autocorrelation of cadherin intensity fluctuations in Cdh3 wild type and rescue embryos. Local maxima in the Cdh3 spatial correlation are indicated as peaks (SI, Section 18). This shows the well-defined spatial periodicity in Cdh3 distribution along the cell-cell junction for both wild type and rescue embryos. D. Spatial autocorrelation of cadherin intensity fluctuations in Cdh3 control (solid lines) and Cdh3 rescue (dashed lines) embryos along a single junction at 5 different time frames. E. Single junction spatial autocorrelation of cadherin intensity fluctuations in Cdh3 mutant and Xdd1 mutant embryos. As compared to wild type and rescue embryos, local maxima in the Cdh3 spatial correlation is highly suppressed. The spatial periodicity of Cdh3 distribution along the cell-cell junction is not seen. F. Spatial autocorrelation of cadherin intensity fluctuations in Cdh3 mutant (solid lines) and Xdd1 mutant (dashed lines) embryos along a single junction at 5 different time frames. G. Mean spatial autocorrelation of Cdh3 intensity fluctuations for Xdd1(black) and Cdh3 mutant (magenta) with functional fits to the decay behavior. Both spatial correlations decay to zero is better fit to a power law. This is evidence for the lack of characteristic spatial scale for the correlation in spatial Cdh3 intensity fluctuations. 


\section{$\underline{\text { References: }}$}

Alt, S., P. Ganguly, and G. Salbreux. 2017. Vertex models: from cell mechanics to tissue morphogenesis. Philos Trans R Soc Lond B Biol Sci. 372.

Angelini, T.E., E. Hannezo, X. Trepat, M. Marquez, J.J. Fredberg, and D.A. Weitz. 2011. Glasslike dynamics of collective cell migration. Proceedings of the National Academy of Sciences. 108:4714-4719.

Bénazéraf, B., P. Francois, R.E. Baker, N. Denans, C.D. Little, and O. Pourquié. 2010. A random cell motility gradient downstream of FGF controls elongation of an amniote embryo. Nature. 466:248-252.

Bi, D., J. Lopez, J.M. Schwarz, and M.L. Manning. 2015. A density-independent rigidity transition in biological tissues. Nature Physics. 11:1074.

Blankenship, J.T., S.T. Backovic, J.S. Sanny, O. Weitz, and J.A. Zallen. 2006. Multicellular rosette formation links planar cell polarity to tissue morphogenesis. Dev Cell. 11:459470.

Brieher, W.M., and B.M. Gumbiner. 1994. Regulation of C-cadherin function during activin induced morphogenesis of Xenopus animal caps. J Cell Biol. 126:519-527.

Butler, M.T., and J.B. Wallingford. 2018. Spatial and temporal analysis of PCP protein dynamics during neural tube closure. Elife. 7.

Cetera, M., L. Leybova, B. Joyce, and D. Devenport. 2018. Counter-rotational cell flows drive morphological and cell fate asymmetries in mammalian hair follicles. Nat Cell Biol. 20:541-552.

Chen, C.S., S. Hong, I. Indra, A.P. Sergeeva, R.B. Troyanovsky, L. Shapiro, B. Honig, and S.M. Troyanovsky. 2015. a-Catenin-mediated cadherin clustering couples cadherin and actin dynamics. J Cell Biol. 210:647-661.

Davidson, L.A. 2017. Mechanical design in embryos: mechanical signalling, robustness and developmental defects. Philos Trans R Soc Lond B Biol Sci. 372.

Fagotto, F., N. Rohani, A.S. Touret, and R. Li. 2013. A molecular base for cell sorting at embryonic boundaries: contact inhibition of cadherin adhesion by ephrin/ Eph-dependent contractility. Dev Cell. 27:72-87.

Fernandez-Gonzalez, R., M. Simoes Sde, J.C. Roper, S. Eaton, and J.A. Zallen. 2009. Myosin II dynamics are regulated by tension in intercalating cells. Dev Cell. 17:736-743.

Fletcher, A.G., F. Cooper, and R.E. Baker. 2017. Mechanocellular models of epithelial morphogenesis. Philos Trans R Soc Lond B Biol Sci. 372.

Guillot, C., and T. Lecuit. 2013. Mechanics of epithelial tissue homeostasis and morphogenesis. Science. 340:1185-1189.

Harrison, O.J., X. Jin, S. Hong, F. Bahna, G. Ahlsen, J. Brasch, Y. Wu, J. Vendome, K. Felsovalyi, C.M. Hampton, R.B. Troyanovsky, A. Ben-Shaul, J. Frank, S.M. Troyanovsky, L. Shapiro, and B. Honig. 2011. The extracellular architecture of adherens junctions revealed by crystal structures of type I cadherins. Structure. 19:244-256.

Hong, S., R.B. Troyanovsky, and S.M. Troyanovsky. 2013. Binding to F-actin guides cadherin cluster assembly, stability, and movement. J Cell Biol. 201:131-143.

Huebner, R.J., and J.B. Wallingford. 2018. Coming to Consensus: A Unifying Model Emerges for Convergent Extension. Dev Cell. 46:389-396.

Kale, G.R., X. Yang, J.M. Philippe, M. Mani, P.F. Lenne, and T. Lecuit. 2018. Distinct contributions of tensile and shear stress on E-cadherin levels during morphogenesis. Nat Commun. 9:5021.

Kegel, W.K., and A. van Blaaderen. 2000. Direct observation of dynamical heterogeneities in colloidal hard-sphere suspensions. Science. 287:290-293. 
Kim, H.Y., and L.A. Davidson. 2011. Punctuated actin contractions during convergent extension and their permissive regulation by the non-canonical Wnt-signaling pathway. J Cell Sci. 124:635-646.

Lawton, A.K., A. Nandi, M.J. Stulberg, N. Dray, M.W. Sneddon, W. Pontius, T. Emonet, and S.A. Holley. 2013. Regulated tissue fluidity steers zebrafish body elongation. Development. 140:573-582.

Lee, C.H., and B.M. Gumbiner. 1995. Disruption of gastrulation movements in Xenopus by a dominant-negative mutant for C-cadherin. Dev Biol. 171:363-373.

Levayer, R., and T. Lecuit. 2013. Oscillation and polarity of E-cadherin asymmetries control actomyosin flow patterns during morphogenesis. Dev Cell. 26:162-175.

Levayer, R., A. Pelissier-Monier, and T. Lecuit. 2011. Spatial regulation of Dia and Myosin-II by RhoGEF2 controls initiation of E-cadherin endocytosis during epithelial morphogenesis. Nat Cell Biol. 13:529-540.

Lieber, A.D., Y. Schweitzer, M.M. Kozlov, and K. Keren. 2015. Front-to-rear membrane tension gradient in rapidly moving cells. Biophys J. 108:1599-1603.

Malmi-Kakkada, A.N., X. Li, H.S. Samanta, S. Sinha, and D. Thirumalai. 2018. Cell Growth Rate Dictates the Onset of Glass to Fluidlike Transition and Long Time Superdiffusion in an Evolving Cell Colony. Physical Review X. 8:021025.

Marmottant, P., A. Mgharbel, J. Käfer, B. Audren, J.-P. Rieu, J.-C. Vial, B. Van Der Sanden, A.F. Marée, F. Graner, and H. Delanoë-Ayari. 2009. The role of fluctuations and stress on the effective viscosity of cell aggregates. Proceedings of the National Academy of Sciences. 106:17271-17275.

Merkel, M., and M.L. Manning. 2017. Using cell deformation and motion to predict forces and collective behavior in morphogenesis. Semin Cell Dev Biol. 67:161-169.

Mongera, A., A. Michaut, C. Guillot, F. Xiong, and O. Pourquié. 2019. Mechanics of Anteroposterior Axis Formation in Vertebrates. Annu Rev Cell Dev Biol. 35:259-283.

Mongera, A., P. Rowghanian, H.J. Gustafson, E. Shelton, D.A. Kealhofer, E.K. Carn, F. Serwane, A.A. Lucio, J. Giammona, and O. Campàs. 2018. A fluid-to-solid jamming transition underlies vertebrate body axis elongation. Nature. 561:401.

Moore, S.W., R.E. Keller, and M.A. Koehl. 1995. The dorsal involuting marginal zone stiffens anisotropically during its convergent extension in the gastrula of Xenopus laevis. Development. 121:3131-3140.

Ninomiya, H., R. David, E.W. Damm, F. Fagotto, C.M. Niessen, and R. Winklbauer. 2012. Cadherin-dependent differential cell adhesion in Xenopus causes cell sorting in vitro but not in the embryo. J Cell Sci. 125:1877-1883.

Rauzi, M., P. Verant, T. Lecuit, and P.F. Lenne. 2008. Nature and anisotropy of cortical forces orienting Drosophila tissue morphogenesis. Nat Cell Biol. 10:1401-1410.

Serwane, F., A. Mongera, P. Rowghanian, D.A. Kealhofer, A.A. Lucio, Z.M. Hockenbery, and O. Campàs. 2017. In vivo quantification of spatially varying mechanical properties in developing tissues. Nat Methods. 14:181-186.

Shi, Z., Z.T. Graber, T. Baumgart, H.A. Stone, and A.E. Cohen. 2018. Cell membranes resist flow. Cell. 175:1769-1779. e1713.

Shindo, A., Y. Inoue, M. Kinoshita, and J.B. Wallingford. 2019. PCP-dependent transcellular regulation of actomyosin oscillation facilitates convergent extension of vertebrate tissue. Dev Biol. 446:159-167.

Shindo, A., and J.B. Wallingford. 2014. PCP and septins compartmentalize cortical actomyosin to direct collective cell movement. Science. 343:649-652.

Sinha, S., A.N. Malmi-Kakkada, X. Li, H.S. Samanta, and D. Thirumalai. 2020. Spatially heterogeneous dynamics of cells in a growing tumor spheroid: Comparison between Theory and Experiments. Soft Matter. 16:5294-5304. 
Solon, J., A. Kaya-Copur, J. Colombelli, and D. Brunner. 2009. Pulsed forces timed by a ratchet-like mechanism drive directed tissue movement during dorsal closure. Cell. 137:1331-1342.

Staddon, M.F., K.E. Cavanaugh, E.M. Munro, M.L. Gardel, and S. Banerjee. 2019. Mechanosensitive Junction Remodeling Promotes Robust Epithelial Morphogenesis. Biophys J. 117:1739-1750.

Stooke-Vaughan, G.A., and O. Campàs. 2018. Physical control of tissue morphogenesis across scales. Curr Opin Genet Dev. 51:111-119.

Strale, P.O., L. Duchesne, G. Peyret, L. Montel, T. Nguyen, E. Png, R. Tampe, S. Troyanovsky, S. Henon, B. Ladoux, and R.M. Mege. 2015. The formation of ordered nanoclusters controls cadherin anchoring to actin and cell-cell contact fluidity. J Cell Biol. 210:333346.

Sun, Z., C. Amourda, M. Shagirov, Y. Hara, T.E. Saunders, and Y. Toyama. 2017. Basolateral protrusion and apical contraction cooperatively drive Drosophila germ-band extension. Nat Cell Biol. 19:375-383.

Truong Quang, B.A., M. Mani, O. Markova, T. Lecuit, and P.F. Lenne. 2013. Principles of Ecadherin supramolecular organization in vivo. Curr Biol. 23:2197-2207.

Vanderleest, T.E., C.M. Smits, Y. Xie, C.E. Jewett, J.T. Blankenship, and D. Loerke. 2018. Vertex sliding drives intercalation by radial coupling of adhesion and actomyosin networks during Drosophila germband extension. Elife. 7.

Wallingford, J.B., L.A. Niswander, G.M. Shaw, and R.H. Finnell. 2013. The continuing challenge of understanding, preventing, and treating neural tube defects. Science. 339:1222002.

Wallingford, J.B., B.A. Rowning, K.M. Vogeli, U. Rothbacher, S.E. Fraser, and R.M. Harland. 2000. Dishevelled controls cell polarity during Xenopus gastrulation. Nature. 405:81-85.

Weeks, E.R., J.C. Crocker, A.C. Levitt, A. Schofield, and D.A. Weitz. 2000. Three-dimensional direct imaging of structural relaxation near the colloidal glass transition. Science. 287:627-631.

Williams, M., W. Yen, X. Lu, and A. Sutherland. 2014. Distinct apical and basolateral mechanisms drive planar cell polarity-dependent convergent extension of the mouse neural plate. Dev Cell. 29:34-46.

Yap, A.S., W.M. Brieher, M. Pruschy, and B.M. Gumbiner. 1997. Lateral clustering of the adhesive ectodomain: a fundamental determinant of cadherin function. Curr Biol. 7:308315.

Yap, A.S., G.A. Gomez, and R.G. Parton. 2015. Adherens Junctions Revisualized: Organizing Cadherins as Nanoassemblies. Dev Cell. 35:12-20.

Yap, A.S., C.M. Niessen, and B.M. Gumbiner. 1998. The juxtamembrane region of the cadherin cytoplasmic tail supports lateral clustering, adhesive strengthening, and interaction with p120ctn. J Cell Biol. 141:779-789.

Zhou, J., H.Y. Kim, and L.A. Davidson. 2009. Actomyosin stiffens the vertebrate embryo during crucial stages of elongation and neural tube closure. Development. 136:677-688. 


\section{Huebner, Malmi-Kakkada : Theory Supplement}

\section{Section 1. Active versus passive vertex dynamics}

We used the Manual Tracking plugin in FIJI to obtain the trajectories of vertex pairs. Individual vertex positions were tracked for a time interval of $400 \mathrm{~s}$ every $2 \mathrm{~s}$. By obtaining the timedependent two-dimensional (2D) vertex co-ordinates $\left(x_{L}, y_{L}\right)$ and $\left(x_{R}, y_{R}\right)$ for the left (L) and right $(R)$ vertices respectively, the net distance travelled by the left $(L)$ vertex is,

$\Delta r_{L}=\sqrt{\left(x_{L}\left(t_{f}\right)-x_{L}\left(t_{0}\right)\right)^{2}+\left(y_{L}\left(t_{f}\right)-y_{L}\left(t_{0}\right)\right)^{2}}$

where $x_{L}\left(t_{f}\right), x_{L}\left(t_{0}\right)$ are the vertex positions at the final $\left(t_{f}\right)$ and initial time $\left(t_{o}\right)$ of measurement respectively. A similar equation with $x_{R}, y_{R}$ applies for the right vertex. The length of the junction is,

$L(t)=\sqrt{\left(x_{R}(t)-x_{L}(t)\right)^{2}+\left(y_{R}(t)-y_{L}(t)\right)^{2}}$

To determine the weight of the contribution of each vertex to junction shortening, we define an activity parameter, $A$, as the ratio of net vertex distance moved to the initial junction length i.e. $A_{L}=\frac{\Delta r_{L}}{L\left(t_{0}\right)}$. Similarly, $A_{R}=\frac{\Delta r_{R}}{L\left(t_{0}\right)}$, for the right vertex. If $A_{L}>A_{R}$, the left vertex is labelled as the 'active' vertex while the right vertex is the 'passive' one, and vice versa if $A_{R}>A_{L}$. Over the time frames that we have analyzed the vertex movement, the median value of $L\left(t_{f}\right) / L\left(t_{0}\right) \sim 0.30$, implying that the junctions have shortened by $\sim 70 \%$ as compared to the initial junction length. Both high time resolution ( $2 \mathrm{~s}$ per frame) and low time resolution (20s per frame) imaging data show the same trend that one of the vertices tend to be active, contributing more to junction shortening (Fig 1B-C, Main Text). We confirm that this observation is not due to the overall translation or rotational motion of the cells as detailed below (Supp. Fig. 2).

\section{Section 2. Normalized junction length dynamics:}

We calculated the normalized cell-cell junction contact lengths to characterize the selfsimilarity in the length change underlying cell neighbor exchanges during convergent extension. We selected all cell-cell contacts that shorten over time intervals $>100$ s, and normalized the change in length as,

$L_{n}(t)=\frac{L(t)-L\left(t_{f}\right)}{L\left(t_{0}\right)-L\left(t_{f}\right)}$

where $L\left(t_{f}\right), L\left(t_{0}\right)$ are the junction lengths at the final and initial time points respectively. The normalized junction length dynamics, $L_{n}(t)$, provides insight into the active processes that underlie vertex movement driving CE. Since junction lengths are highly heterogeneous (Supp. Fig $4 \mathrm{~A}$ ) relative to, $L\left(t_{0}\right)$, and the time to closure, $t_{f}-t_{0}$, the normalization in Eq. (3) allows us to rescale all the length changes to values between 1 and 0 . The normalized length curve was smoothed (over 10-time frame windows $=20 \mathrm{~s}$ ) to remove higher frequency noise. To determine if junction shortening exhibits a self-similar behavior across multiple embryos, we rescaled the time axis in $L_{n}(t)$ by the relaxation time $\tau_{f}$, defined as the time at which $L_{n}(t=$ 
$\left.\tau_{f}\right)=0.3$. This corresponds to a $70 \%$ reduction in the junction length. Rescaling the time axis by $t / \tau_{f}$ collapses the normalized lengths onto the functional form, $L_{n}\left[t / \tau_{f}\right]=e^{-1.5\left(\frac{t}{\tau_{f}}\right)^{3.8}}$

which is a single compressed exponential (Supp. Fig. 4B). The extent of the self-similarity is striking in comparison to both non-shortening (Fig $2 \mathrm{H}$, Main Text) and cis-mutant normalized junction lengths (Fig 6D, Main Text). Notice that for $t<\tau_{f}$, change in normalized junction length is slower than exponential decay. However, for $t>\tau_{f}$, normalized junction length significantly shortens faster than would be predicted based on exponential decay. Therefore, the compressed exponential behavior for $L_{n}$ provides evidence that the persistence of junction shortening increases with time.

\section{Section 3. Junction length fluctuations}

To analyze the instantaneous change in the junction length, we calculated, $\delta L(t)=(L(t)-$ $L(t+\delta t))$, where $\delta t=2 s$ and $t$ is the time. The unit of the length fluctuations is $\mu m$. When the junction shortens, $\delta L(t)>0$, while extension implies $\delta L(t)<0$ (Fig. 4E, Main Text).

\section{Section 4. Quantifying the heterogenous dynamics of vertices: Mean Square Displacement (MSD), van Hove function and the velocity autocorrelation.}

The characteristics of vertex dynamics could provide clues as to the active mechanisms that promote or impede vertex movement. An important parameter to quantify vertex dynamics is the Mean Square Displacement (MSD), as a function of the lag time t. Time averaged MSD, $\bar{\Delta}(t)_{i}$, is calculated using the vertex positions $\vec{r}_{i}\left(t^{\prime}\right)$,

$\bar{\Delta}(t)_{L, i}=\frac{1}{T-t} \int_{0}^{T-t}\left|\vec{r}_{L, i}\left(t^{\prime}+t\right)-\vec{r}_{L, i}\left(t^{\prime}\right)\right|^{2} d t^{\prime}$

where $T=400 \mathrm{~s}$ and subscript $L$ stands for the left vertex. Taking the average over $\mathrm{N}$ independent vertex trajectories, labelled by the index $i$, we obtain the ensemble averaged MSD, $\Delta(t)_{L}=\frac{1}{N} \sum_{i=1}^{N} \bar{\Delta}(t)_{L, i}$. The same procedure is used to calculate the MSD for the right vertex (see Fig 1E, Main Text). In many physical systems the MSD increases with a power law, i.e. $\Delta(t) \sim t^{\alpha}$. When the vertex motion is uncorrelated in time and along random directions, the dynamics is described as Brownian, and the MSD exponent is unity, $\alpha=1$. Sub-diffusive, $\alpha<1$, movement occurs when there is a hindrance to motion or the dynamics is highly correlated. For example, when a particle in caged by its immediate neighbors, sub-diffusive motion results. Super-diffusive MSD, $\alpha>1$, is seen when the motion is highly directed.

We found substantial heterogeneity in the individual vertex MSD as seen from the plot of $\bar{\Delta}(t)_{i}$ (Suppl. Fig 2A). Active and passive vertex MSDs span 3 orders of magnitude of time lag. Two distinct time regimes are observed for both active and passive vertex movements: (i) at short time lags, $t<30 s$, active and passive vertex movements are random, characterized by MSD exponent $\alpha \sim 1$. (ii) For $t>40$ s, active vertices show strong superdiffusive movement while 
passive vertices undergo a slowdown followed by a recovery towards superdiffusive motion (see Fig 1E, Main Text). These distinct differences between active versus passive vertices are observed in the ensemble averaged MSD, $\Delta(t)$ for 20 vertices from 10 different embryos.

To eliminate the effect of translation and rotational motion of the entire tissue, we tracked vertex positions with respect to the center of an egg yolk particle (Suppl. Fig 2C) typically present within cells as well as nearby stationary vertices (Suppl. Fig 2E). In this manner, we analyzed the relative vertex positions, $\vec{r}_{r e l}$, with respect to a frame of reference within the tissue being imaged. By extracting the co-ordinates of the center of an egg yolk within a cell or nearby vertices, $\vec{r}_{c}$, we obtain the relative vertex positions, $\vec{r}_{r e l, L}=\vec{r}_{L}-\vec{r}_{c}$. We then evaluated the mean square relative displacements (MSRD) for the left and right vertex pairs using Eq. (5) above (Suppl. Fig 2D,F). The distinct differences between active versus passive vertex dynamics is conserved in this relative co-ordinate system, indicating that the asymmetry in active versus passive vertex movement is not due to translational or rotational motion of the whole tissue (see Suppl. Fig 2).

Van Hove Function: Insights into vertex motion may be obtained by analogy to spatially heterogenous dynamics in supercooled liquids [1,2]. The distribution of particle displacements is expected to be a Gaussian in simple fluids. In supercooled liquids, however, the displacements of a subset of particles deviate from the Gaussian distribution [1]. From the distance moved by a vertex during the time interval $\delta t$, defined as $\left|\delta r_{i}(\delta t)\right|=\mid \vec{r}_{i}(t+\delta t)-$ $\vec{r}_{i}(t) \mid$, the van Hove function for vertex displacement (or the probability distribution of vertex step size) is,

$P(\delta r \mid \delta t)=\left\langle\frac{1}{N} \sum_{i=1}^{N} \theta\left(\delta r_{i}-\left|\vec{r}_{i}(t+\delta t)-\vec{r}_{i}(t)\right|\right)\right\rangle$

where the average is over $\mathrm{N}$ independent vertex trajectories. The van Hove distribution at $\delta t=$ $40 s$, for active (red) and passive vertices (blue) is shown in Suppl. Fig 3A. The $40 s$ time interval is long enough to clearly observe the differences in the distances moved by active and passive vertices. The van Hove distribution at $\delta t=4 s$ is shown in Suppl. Fig 3B. At this short time interval, distances moved by active and passive vertices are similar and is well fit by a Gaussian (see inset Suppl. Fig 3B). However, the van Hove distribution deviates significantly from the Gaussian distribution at $\delta t=40$ s (see inset Suppl. Fig 3A), indicating the growing heterogeneity in the vertex displacements.

Average velocity distribution and velocity autocorrelation function (VACF):

To further quantify the striking differences in the movement of active and passive vertices, we calculate the average velocity of the vertices. The average velocity over a time interval $\tau$ is defined as,

$\vec{v}_{L}(\tau)=\frac{\vec{r}_{L}(t)-\vec{r}_{L}(t+\tau)}{\tau}$

Replacing $\vec{r}_{L}$ by $\vec{r}_{R}$ gives the average velocity of the right vertex. We analyze the average velocity over a time interval $\tau$ because experimental data is also an average over the time 
resolution of the iSIM microscope. We then compare the speed distribution $\left(\left|\vec{v}_{L}(\tau)\right|\right)$ of active and passive vertices over both short, $\tau=4 s$ (Suppl. Fig 3C, blue for passive and red for active vertices) and longer time intervals, $\tau=60 \mathrm{~s}$ (Suppl. Fig 3D). At the smaller time interval, $\tau=4 s$, the speed distribution of active and passive vertices are similar. This indicates minimal differences between active and passive vertex dynamics at short time scales. The difference in active and passive speed distribution is, however, pronounced at $\tau=60 \mathrm{~s}$. The passive vertex speed distribution peaks at a smaller value and decays rapidly for larger speed values, compared to active vertices. This illustrates the fluidization in the movement of active vertices that develops over a time scale of order $50 \mathrm{~s}$ in agreement with other measures such as the MSD and the van Hove distribution as reported above.

To probe the time interval over which the average velocity (at fixed $\tau$ ) is correlated with average velocity at a time point separated by $\delta t$, we calculate the velocity autocorrelation function (VACF),

$C_{v}^{\tau}(\delta t)=\langle\vec{v}(t+\delta t) \cdot \vec{v}(t)\rangle$

where the average is defined as $\langle\ldots\rangle=\frac{1}{T-\delta t} \int_{0}^{T-\delta t} \ldots d t$. The VACF is normalized such that $C_{v}^{\tau}(\delta t=0)=1$. At the shorter time interval of $\tau=4 s$, VACF for active and passive vertices exhibit a rapid decay to zero (Suppl. Fig 3E, blue for passive and red for active vertices). Individual vertex VACF are plotted in transparent colors and the mean as dashed lines (blueblack dashed line for passive vertices and red-black dashed line for active vertices). Analyzing vertex velocities at $\tau=60$ s clearly brings out the different dynamics that characterize active versus passive vertices (Suppl. Fig 3F, blue for passive and red for active vertices). Velocity correlations decay quicker for passive vertices, becoming negative and then rebounds. However, active vertex velocity correlations are more persistent with time as evident from the longer time to decay.

\section{Section 5. Self-overlap parameter and dynamic heterogeneity}

To quantify the highly asymmetric vertex movement that underlies $\mathrm{CE}$, we measured the fractional change in vertex positions over a time interval t using the self-overlap order parameter, defined as:

$\langle Q(t)\rangle=\frac{1}{N} \sum_{i}^{N} w_{i}$

where $w_{i}=1$ if $\left|\vec{r}_{i}\left(t^{\prime}+t\right)-\vec{r}_{i}\left(t^{\prime}\right)\right|<L_{c}$ and $w_{i}=0$ otherwise. The self-overlap parameter is dependent on the length scale that is probed by $L_{c}$ and represents the probability that vertices have moved by a specified length scale over a time interval, $\mathrm{t}$. We chose $L_{c}=1.3 \mu \mathrm{m}$, as this is the distance scale over which movement of active and passive vertex become distinct. This is evident from the plot of MSD (Fig 1E, Main Text) for active and passive vertices where the dynamics begins to differ at a length scale of $>1 \mu \mathrm{m}$. If a vertex moves less than $L_{c}=1.3 \mu \mathrm{m}$ over the time interval $t$, the vertex is considered to have $100 \%$ overlap with its previous position, and hence assigned a value 1 . However, if the vertex has moved more than $1.3 \mu \mathrm{m}$ 
within the time interval $t$, we consider this as $0 \%$ overlap. The self-overlap function, $\langle Q(t)\rangle$, is calculated by averaging over a range of initial times, $t^{\prime}$, followed by ensemble averaging over individual vertices (Supp. Fig. 3G-H). The active vertex self-overlap function decays rapidly and can be fit to a single exponential decay function, indicating liquid like dynamics. However, passive vertex overlap function shows a two-step decay, a signature of glass-like dynamics (Supp. Fig. 3G).

Although the MSD and the self-overlap function $\langle Q(t)\rangle$ are useful to quantitatively characterize vertex movement, other metrics are needed to gather further insights into the dynamic heterogeneity and correlations in vertex movement that emerge temporally during CE. In systems approaching the glass transition, the cooperativity of motion increases such that the length and time scales characterizing the dynamic heterogeneity are expected to grow sharply. In supercooled liquids, the fourth order susceptibility, $\chi_{4}(t)$, provides a unique way to distinguish the dynamic fluctuations between liquid and frozen states [3]. Therefore, we compute the fourth order susceptibility from the variance of the self-overlap parameter, $\chi_{4}(t)=\left\langle Q(t)^{2}\right\rangle-\langle Q(t)\rangle^{2}$

Similar to structural glasses, the dynamic heterogeneity, quantified by $\chi_{4}(t)$ increases with time, peaks at a maximum time interval, $t_{M}$ and then decays (Supp. Fig. $3 \mathrm{I}$ ). The dynamic heterogeneity is manifested as dramatic variations between individual vertex trajectories in both active and passive vertex movements. For active vertices, $\chi_{4}(t)$ peaks at $t_{M} \sim 120 \mathrm{~s}$ while for passive vertices heterogeneity peaks at a longer time interval $t_{M} \sim 170 s$ (Supp. Fig. 3I). The time scale associated with the peak in dynamic heterogeneity is consistent with the viscoelastic relaxation time (further discussed below), known to be the characteristic relaxation time for vertices connected by the cell cortex under tension [4]. For non-shortening junctions, $\chi_{4}(t)$, does not show a peak (Suppl. Fig 3J). We anticipate the peak to be at a much longer time scale for vertices of non-shortening junctions.

\section{Section 6. Cadherin clustering from the spatial autocorrelation function}

To determine the characteristic spatial correlation of cadherin intensity fluctuations, we analyze the pixel-by-pixel Cadherin3 (Cdh3) intensity data, $I\left(\vec{r}_{i}\right)$, along the medio-lateral cell-cell interface ( $v$-junction). Here, $\vec{r}_{i}$ is the position of the i-th pixel in the iSIM image. The spatial autocorrelation function of the cadherin intensity fluctuations as a function of distance, $r$, along the cell-cell interface is,

$C(r)=\sum_{i, j} \theta\left(r-\left|\vec{r}_{i}-\vec{r}_{j}\right|\right)\left[\frac{\left(I\left(\vec{r}_{i}\right)-\langle I\rangle\right)\left(I\left(\vec{r}_{j}\right)-\langle I\rangle\right)}{\left\langle I^{2}\right\rangle-\langle I\rangle^{2}}\right]$,

where $\theta(z)=1$ if $z=0, \theta(z)=0$ for any other value of $z$. $\langle I\rangle$ is the mean cadherin intensity over all the pixels along the cell-cell junction. $C(r)$ is normalized such that $C(r=0)=1$. The cadherin correlation length is defined as the distance, $\xi$, at which $C(r=\xi)=0$. This provides a measure of the distance scale at which the correlation in cadherin intensity fluctuations is lost. Equivalently, $\xi$, sets the spatial persistence of cadherin fluctuations along the cell-cell junction, providing a quantitative measure of lateral cadherin clustering. We analyzed cadherin clustering 
patterns along individual cell-cell junctions separately at time intervals of $2 \mathrm{~s}$ and obtained the spatial correlation behavior for individual junctions from 10 embryos. The mean of the cadherin spatial correlation (over 100s of time points) for wild type embryos is reported in Fig. 4C, Main Text with the error bar denoting the standard deviation. To analyze the dynamic variation in cadherin cluster size as a function of time, $C(r)$, was calculated over a time interval of 320s at 2 s resolution. The fluctuation in cluster size is given by, $\delta \xi(t)=\xi(t)-\langle\xi\rangle_{t}$, where $\langle\xi\rangle_{t}$ is the mean cluster size over the analyzed time interval (see Fig. 4D Main Text). The cluster size fluctuation, $\delta \xi(t)$, was smoothed (over 10-time frame windows $=20$ s) in order to remove high frequency noise.

\section{Section 7. Cross-correlation between cadherin cluster size and the junction length fluctuations}

The normalized cross-correlation between junction length fluctuations, $\delta L(t)$, and cadherin cluster size fluctuations, $\delta \xi(t)$, was calculated in MATLAB using,

$C_{\delta L, \delta \xi}(\tau)=\int_{0}^{T} \delta \xi(t) \times \delta L(t+\tau) d t$,

where $\mathrm{T}$ is the total time of analysis and $\tau$ is the lagtime. We analyzed the cross-correlation for 18 junction shortening events and show the correlation coefficient as a heatmap in Fig 4F Main Text.

\section{Section 8. Asymmetry in cadherin clustering:}

To quantify the asymmetry in Cdh3 clustering in the spatial region near the left and right vertices, we calculated the spatial correlation in cadherin intensity fluctuations, $C(r)$ (see Eq. (11)), in a region spanning $3.25 \mu \mathrm{m}$ adjacent to left and right vertices. The spatial region is chosen such that on average it is $3 X$ larger than typical cadherin cluster size of order $1 \mu \mathrm{m}$. The localized cadherin clustering behavior adjacent to active and passive vertices, quantified by the spatial correlation in cadherin intensity fluctuations, is shown in Fig. 4G Main Text. Fig 4G, Main Text also compares the local cadherin clustering behavior in non-shortening junctions to shortening junctions.

We then wondered if spatial correlation in cadherin expression is stable in time across a single cell-cell junction. We computed the spatial cadherin correlation at 2 second time intervals along single cell-cell junctions from multiple embryos. Our results indicate that the spatial correlation length of cadherin expression is highly heterogeneous in time, with the correlation length varying from $0.2 \mu \mathrm{m}$ to $1.6 \mu \mathrm{m}$, as shown in Supp. Fig. 6. To decipher how the spatial correlation in cadherin expression along the cell-cell junction varies with time near active and passive vertices, we present the data for spatial autocorrelation of cadherin fluctuations in Supp. Fig. 6A-J. In each panel, individual blue (red) lines correspond to the spatial correlation in cadherin fluctuations near passive (active) vertices. The difference in spatial cadherin correlation between active and passive vertices in shortening junctions are shown in Supp. Fig. 6A-J whereas the same data for non-shortening junctions are shown in Supp. Fig. 6K-Q. By extracting the length of the spatial 
correlation as discussed in Section 6 above, the summary of the cadherin spatial correlation data is presented in the Main Text Fig. 4G.

We used an alternative definition of $\mathrm{C}$-cadherin cluster size to confirm our results. By fitting the decay in the cadherin spatial autocorrelation function to zero by an exponential function, we can extract the cluster size. We find that the asymmetry in the local cadherin clustering behavior is independent of the definition of the cluster size (Suppl. Fig 5B-D). Hence, we conclude that cadherin clustering is enhanced near active vertices as opposed to passive vertices in shortening junctions (Fig. 4G Main Text, Supp. Fig. 5C) while it is symmetric near left and right vertices in non-shortening junctions (Supp. Fig. 5D).

\section{Section 9. Theoretical Model}

Vertex based models are important for studying the dynamics of confluent cell layers [5]. The junction between three or more cells (vertices) are represented as point particles. The connecting edge between vertices represent cell-cell interfaces. We developed a theoretical model for junction shortening to understand the asymmetric dynamics of vertices. Our model, shown in Fig 2A-B Main Text, is a coarse-grained representation of a collection of cells intercalating mediolaterally. Each vertex, bounding the v-junction, are connected to Maxwelllike components with viscous and elastic elements. Elastic properties are modeled by springs with stiffness, $k$, and actuators characterize the viscous motion of cell vertices (see Fig 2A Main Text; $\gamma$ is the viscosity). For the purposes of visualization, we depict the spring-actuator element as being in the direction away from the cell-cell interface, exerting a compressive force on the vertices. This need not be the case as the forces and mechanical factors contributing to junction shortening can also be localized within the cell-cell junction. For the purposes of simplicity in visualization, we have picked a direction for the spring-actuation element.

We assume that the position of the left vertex, $\vec{r}_{L}\left(\stackrel{\text { def }}{=} x_{L}, y_{L}\right)$, evolves according to the equation of motion:

$\frac{d \vec{r}_{L}}{d t}=-\frac{\vec{k}_{L} \cdot \vec{r}_{L}}{\gamma_{L}}+\frac{\vec{F}_{L}}{\gamma_{L}}+\zeta_{L}$

where $\vec{k}_{L}$ is the elasticity of the left $(L)$ vertex, $\vec{F}_{L}$ is the contractile force responsible for viscous deformation of the vertex and $\gamma_{L}$ is viscosity coefficient of the vertex. Replacing the subscript $L$ with $R$ above gives the equation of motion for the right vertex. The local elasticity near the vertices are accounted for by a connected harmonic spring with strength $\vec{k}_{L}$. The spring is connected in series with an actuator that supplies the contractile force, $\vec{F}_{L}$. It is likely that the noise in a physical or biological system is correlated in time. Consistent with our observation that fluctuations in junction length are correlated in time, we model $\zeta_{L}$ as the colored noise experienced by the vertices. The noise, $\zeta_{L}$, represents the coupling of the vertices to their immediate local environment, satisfying $\left\langle\zeta_{L}(t) \zeta_{L}(s)\right\rangle=A e^{-|t-s| / \tau_{n}}$ with the mean $\left\langle\zeta_{L}(t)\right\rangle=0$. The coefficient, $A$, is the noise strength. For large noise strength, vertex positions show large amplitude deviations from the position dictated by the minimum of the elastic force, as 
constrained by the spring. For small persistence time of the correlated noise, $\tau_{n}$, the vertex dynamics is highly uncorrelated in time. At large persistence times, however, the noise induced fluctuations in the vertex positions are correlated over the timescale $\tau_{n}$. We set the noise correlation time to be the persistence time of junction length fluctuations. The colored noise satisfies, $\frac{d \zeta_{L}}{d t}=-\frac{\zeta_{L}}{\tau_{n}}+\frac{1}{\tau_{n}} \eta(t)$, where $\eta(t)$ is the Gaussian white noise source characterized by delta correlation $\langle\eta(t) \eta(s)\rangle=\delta(t-s)$ and mean $\langle\eta\rangle=0$.

Since the movement of vertices along the medio-lateral direction is much more persistent as opposed to the perpendicular direction, as evident from the closure of junctions, we simplify the model to consider only one-dimensional (1D) motion. Henceforth, we drop the vector notation and focus on the vertex dynamics along the $x$-axis.

By considering the basic vertex equations in the Langevin picture,

$\frac{d x_{L}}{d t}=-\frac{k_{L}}{\gamma_{L}} \times\left(x_{L}-a_{L} t^{\psi_{L}}\right)+\zeta_{L}$

$\frac{d x_{R}}{d t}=-\frac{k_{R}}{\gamma_{R}} \times\left(x_{R}-\left(L_{0}-a_{R} t_{R}\right)\right)+\zeta_{R}$

we model the vertex equations of motion in analogy to particles moving in a translating optical trap. The minimum of the left elastic 'trap' changes dynamically due to the term $a_{L} t \psi_{L}$ in Eq. (14) (modeled by the left actuator). Similarly, the right elastic 'trap' is translated from its initial position $L_{0}$ by $a_{R} t^{\psi_{R}}$ in Eq. (15) (modeled by the right actuator). These terms serve as a proxy for active contractile forces which viscously deform the cell edges. Hence, we refer to the exponents, $\psi_{L}$ and $\psi_{R}$, as the rest length exponents. The physical implication of the rest length exponent is that the rest length of the junction varies dynamically. The contractile force is, $F_{L, \text { total }} \stackrel{\text { def }}{=}-V^{\prime}\left(x_{L}\right)$ in Eq. (13), where $V\left(x_{L}\right)$ is the time dependent 'trap' potential of the form $V_{L}=0.5 k_{L}\left(x_{L}-a_{L} t_{L}\right)^{2}$ and $V_{R}=0.5 k_{R}\left(x_{R}-\left(L_{0}-a_{R} t_{R}\right)\right)^{2}$. The stochastic movement of the vertices in a translating potential leads to a ratchet-like effect where the vertex dynamics has a specified direction. This directionality in the motion of the vertices does not arise, however, from the asymmetry in the potential but rather from the asymmetric translation of the potential well minimum or the dynamic rest length.

Hence, the active time dependent forces contributing to junction shortening were modeled in silico as,

$$
F_{L}(t)=k_{L} a_{L} t^{\psi_{L}}
$$

$F_{R}(t)=k_{R}\left(L_{0}-a_{R} t^{\psi_{R}}\right)$

where $a_{L}$ and $a_{R}$ are the 'acceleration' of the left and right vertices respectively, and the exponents $\psi_{L}$ and $\psi_{R}$ determine the temporal dynamics of the contractile force. We include the acceleration term to account for the experimentally observed increase in the persistence of junction shortening as a function of time (see Fig 2G Main Text; See also Sec. 2 above). The initial condition is set as $x_{L}(t=0)=0$, and $x_{R}(t=0)=L_{0}$, with $L_{0}$ being the initial cell-cell junction length. We arbitrarily assign the left side to be active, with the time dependent active force rising in proportion to $t^{\psi_{L}}\left(\psi_{L}>\psi_{R}\right)$. The right side is assigned to be passive, with force increasing with time as $\propto t^{\psi_{R}}$. The difference in the rest length exponents, $\psi_{L}$ versus $\psi_{R}$, determines which vertex is active. 
The equations of motion then become:

$$
\begin{aligned}
\frac{d x_{L}}{d t} & =-\frac{k_{L}}{\gamma_{L}} x_{L}+\frac{k_{L} a_{L} t^{\psi_{L}}}{\gamma_{L}}+\zeta_{L} \\
\frac{d x_{R}}{d t} & =-\frac{k_{R}}{\gamma_{R}} x_{R}+\frac{k_{R}\left(L_{0}-a_{R} t^{\left.\psi_{R}\right)}\right.}{\gamma_{R}}+\zeta_{R}
\end{aligned}
$$

Defining $\bar{x}_{L}=\frac{x_{L}}{x_{0}}, \bar{t}=\frac{t}{\tau}$ and $\bar{a}_{L}=\frac{a_{L}}{a_{L}^{0}}$, where $x_{0}=10 \mu m, \tau=10^{2} \sec$ and $a_{L}^{0} \tau \psi_{L}=x_{0}$, we recast the equations of motions into dimensionless forms. Similar normalization with $L$ replaced by $\mathrm{R}$ applies for the right vertex. The system of equations is scaled with the characteristic length and time, $x_{0}$ and $\tau$, physiologically relevant for cells undergoing convergent extension. In terms of the normalized quantities, the equation of motion is,

$\frac{d \bar{x}_{L}}{d \underline{t}}=-\frac{1}{\bar{\tau}_{L}} \times\left(\bar{x}_{L}-\bar{a}_{L} \bar{t} \psi_{L}\right)+\bar{\zeta}_{L}$

$\frac{d \bar{x}_{R}}{d \bar{t}}=-\frac{1}{\bar{\tau}_{R}} \times\left(\bar{x}_{R}-\left(\bar{L}_{0}-\bar{a}_{R} \bar{t}^{\psi_{R}}\right)\right)+\bar{\zeta}_{R}$

where the parameter $\frac{k_{L}}{\gamma_{L}} \stackrel{\text { def }}{=} \frac{1}{\tau_{L}}$ has the dimension of inverse time $\frac{1}{s}$. When normalized by the characteristic timescale $\tau, \frac{\tau}{\tau_{L}}=\tau \times\left(\frac{k_{L}}{\gamma_{L}}\right)=\frac{1}{\bar{\tau}_{L}}$, we obtain a dimensionless parameter which we refer to as the viscoelastic ratio.

\section{Section 10. Dynamic rest length and colored noise}

In vertex-based models for plant cells, the cell-cell interface length is modeled with a spring having a characteristic rest length [6]. Any deviation in the length of the cell-cell interface from the rest length is energetically unfavorable. In vertex models for animal cells, such a rest length is typically not included [5]. In our coarse-grained vertex model, we include a spring term with dynamic rest lengths. We show that this model accounts for the asymmetric vertex dynamics and quantitative experimental features of the junction shortening behavior. By studying actomyosin contractility in combination with theoretical modeling, it has recently been shown that epithelial junctions exhibit both elastic and viscous remodeling behavior [7].

The existence of memory effects in junction shortening necessitates the addition of the colored noise term. Previous vertex-based models have considered random white noise indicating no memory effect. However, by experimentally quantifying the junction length fluctuations, we would like to point out that colored noise may be important to consider in modeling biological systems.

\section{Section 11. Parameter values for elasticity and viscosity:}

The viscoelastic ratios, $1 / \bar{\tau}_{L}$ and $1 / \bar{\tau}_{R}$,were varied from 0.05 to 5 equivalent to $5 \times 10^{-4} S^{-1}-$ $0.05 s^{-1}$ in dimensional units. Therefore, the viscoelastic relaxation time is in the range of 
$20 s-2000 s$. Spring stiffness, $k$, in the range between $100 \mathrm{pN} / \mu \mathrm{m}$ and $1 \mathrm{nN} / \mu \mathrm{m}[8,9]$ and the viscosity, $\gamma \sim 100 n N$. s / $\mu m$ [10], accounts for the elastic and viscous properties of tissues previously reported in the literature. For these values, one obtains the viscoelastic relaxation time in the range of $1 s-100 s$. Therefore, the viscoelastic ratio used in our model is within an order of magnitude of the physiological values for both tissue stiffness and viscosity.

\section{Section 12. Simulation Details:}

We consider a wide range of values for both the viscoelastic ratio and the rest length exponent for the active vertex, $\psi_{L}$. The time step in the simulation is $\Delta t=0.0022=0.22 \mathrm{~s}$, chosen to be smaller than the characteristic viscoelastic relaxation time (of order $10 \mathrm{~s}$ ). We evolve the simulation for a total of $n=20,000$ steps $(4,400 s$ in real units). The equations of motion are solved using the Euler method for each vertex. If at any point during the simulation, the left and right vertex positions approach one another to a distance less than $0.5 \mu \mathrm{m}$, we label the junction as having successfully completed the shortening. The initial junction length was set to be $L_{0}=2$, equal to $20 \mu \mathrm{m}$ in real units. The range of rest length exponents we consider is limited by the need to ensure that the minima of the potentials do not overlap during a given simulation run. To generate the phase diagram for the probability of junction shortening as a function of the rest length exponent and the viscoelastic ratio, we consider for the left active vertex $0.05 \leq \frac{1}{\bar{\tau}_{L}} \leq 5$ at intervals of 0.5 . Rest length exponents in the range, $1.7<\psi_{L}<2$, were simulated at intervals of 0.25 for the active vertex. $\psi_{R}=1.3$ is fixed for the passive right vertex. The acceleration of the potential minima, is set to be $\bar{a}_{L}=\bar{a}_{R}=0.001$. The viscoelastic ratio for the right passive vertex is fixed at $\frac{1}{\bar{\tau}_{R}}=0.1$. We simulated 100 junction shortening events at each value of the parameters $\frac{k_{L}}{\gamma_{L}}$ and $\psi_{L}$. By monitoring the percent of successful junction shortening events, we generate the phase diagram in Fig 2C (Main Text).

\section{Section 13. Effect of viscoelasticity on the shortening of junctions:}

We observe in the phase diagram (Fig 2C, Main Text) that at a fixed value of the rest length exponent, modulating the asymmetry of the viscoelastic parameter $\left(\left(\frac{k_{L}}{\gamma_{L}}\right) /\left(\frac{k_{R}}{\gamma_{R}}\right)\right)$ leads to a transition from non-shortening (failure to shorten) to junction shortening (successful shortening) regime. At constant $\psi_{L}=1.95$, for low values of the active vertex viscoelastic parameter, $\left(\bar{\tau}_{R} / \bar{\tau}_{L}\right)<6.9$, less than $40 \%$ of the junctions shorten. However, at higher values of the viscoelastic parameter, $\bar{\tau}_{R} / \bar{\tau}_{L}>15$, more than $80 \%$ of the junctions successfully execute shortening. Therefore, the theory predicts that the local viscoelasticity is critical for cells to intercalate medially and effect convergent extension. We calculate the normalized length for non-shortening junctions and found that the self-similarity in junction length dynamics is broken, in agreement with experimental results (Fig. $2 \mathrm{H}$, Main Text). $L_{n}$ for non-shortening junctions is characterized by large fluctuations away from the expected compressed exponential behavior, as quantified by the residue (see details below). Simulated junction 
length dynamics for the non-shortening case (grey curves in Fig $2 \mathrm{H}$ Main Text) is obtained for parameter values $1 / \bar{\tau}_{L}=0.05,1 / \bar{\tau}_{R}=0.05$ and $\psi_{L}=2, \psi_{R}=1.3$. Meanwhile, for the shortening phase (grey curves in Fig 2D Main Text), $1 / \bar{\tau}_{L}=5,1 / \bar{\tau}_{R}=0.05$ and $\psi_{L}=2, \psi_{R}=$ 1.3. Therefore, asymmetry in viscoelasticity is critical for junctions to execute shortening. Our model points out that the persistent dynamics of active vertices, enabling the efficient shortening of the cell-cell interfaces, is a direct consequence of the faster viscoelastic relaxation time.

\section{Section 14. Residue}

We quantify the deviation of the normalized junction shortening from the expected compressed exponential behavior by calculating the rescaled time, $t_{r}=\frac{t}{\tau_{f}}$, and $\omega=$ $\left|e^{-1.5\left(t_{r}\right)^{3.8}}-L_{n}\right|$, where $\omega$ is the residue. $\tau_{f}$, is defined as the time at which $L_{n}\left(t=\tau_{f}\right)=0.3$. In Fig 2I (Main Text), non-shortening junctions show strong deviations from the expected compressed exponential behavior while shortening junctions closely follow the compressed exponential form.

\section{Section 15. Alternative form of the contractile force}

To test the robustness of the conclusions obtained using our model, we consider an alternative form of the contractile force experienced by the vertices. We model the actuators contributing to viscous junction shortening as moving with constant velocities $-\bar{v}_{L}$ and $\bar{v}_{R}$ - for the left and right vertices respectively:

$$
\begin{aligned}
& \frac{d \bar{x}_{L}}{d \bar{t}}=-\frac{1}{\bar{\tau}_{L}} \times\left(\bar{x}_{L}-\bar{v}_{L} \bar{t}\right)+\bar{\zeta}_{L} \\
& \frac{d \bar{x}_{R}}{d \bar{t}}=-\frac{1}{\bar{\tau}_{R}} \times\left(\bar{x}_{R}-\left(\bar{L}_{0}-\bar{v}_{R} \bar{t}\right)\right)+\bar{\zeta}_{R}
\end{aligned}
$$

The 'trap' potential in this scenario is of the form, $V_{L}=k_{L}\left(x_{L}-v_{L} t\right)^{2}$ and $V_{R}=$ $k_{R}\left(x_{R}-\left(L_{0}-v_{R} t\right)\right)^{2}$, moving with constant velocities. Left vertex is defined to be active with $\bar{v}_{L}>\bar{v}_{R}$. The velocity is normalized as $\bar{v}_{L}=v_{L} /\left(\frac{x_{0}}{\tau}\right)$. The passive vertex velocity is fixed at $\bar{v}_{R}=$ 0.011 , which in dimensional units correspond to $0.0011 \mu \mathrm{m} / \mathrm{s}$. The active vertex velocity is varied in the range of $0.03 \leq \bar{v}_{L} \leq 0.034$, which in dimensional units is between $0.003 \mu \mathrm{m} / \mathrm{s}$ $0.0034 \mu \mathrm{m} / \mathrm{s}$. Experimental vertex shortening velocities in the range of $0.001 \mu \mathrm{m} / \mathrm{s}$ to $0.021 \mu \mathrm{m} / \mathrm{s}$ was reported by some of us in a previous work [11]. Fixing the passive viscoelastic ratio at, $1 / \bar{\tau}_{R}=0.1$, we varied $0.05 \leq 1 / \bar{\tau}_{L} \leq 5$ for the active vertex. Keeping all the other parameters the same, we arrive at the same conclusion that local junction viscoelastic response is critical to effect junction shortening (Suppl. Fig 4D). Therefore, our conclusions are not affected by the specific form of the vertex dynamics. A crucial aspect is that the two potential well minima should move asymmetrically in time. 


\section{Section 16. Transverse fluctuations of the vertices}

We quantify the intermittent movement (see Fig 3A Main Text) of the vertices perpendicular to the motion that contributes to the junction shortening by calculating the transverse fluctuations, $R_{T}$. The transverse step size is given by, $\delta r_{T}(t)=\left|\delta \vec{r}_{L}(t)\right| \sin (\theta)$, where $\delta \vec{r}_{L}(t)=$ $\vec{r}_{L}(t)-\vec{r}_{L}(t-\delta t)$ and the angle $\theta$ is the obtained from the dot product, $\delta \vec{r}_{T} \cdot \Delta \vec{r}_{L}=$ $\left|\delta \vec{r}_{T}\right|\left|\Delta \vec{r}_{L}\right| \cos (\theta)$. Here, the net displacement of the Left(L) vertex is given by, $\Delta \vec{r}_{L}=$ $\left(x_{L}\left(t_{f}\right)-x_{L}\left(t_{0}\right)\right) \hat{x}+\left(y_{L}\left(t_{f}\right)-y_{L}\left(t_{0}\right)\right) \hat{y}$. Similar equation applies for the right vertex with $x_{L}, y_{L}$ replaced by $x_{R}, y_{R}$. To better quantify the intermittent dynamics, we compute the transverse "hop" function, $R_{T}(t)=\left(\delta r_{T}(t)-\left\langle\delta r_{T}\right\rangle_{B}\right)^{2}$

The angular bracket above $\langle. .\rangle_{B}$ denote the average over the time window $B \stackrel{\text { def }}{=}$ $[t-\delta t, t+\delta t]$. We chose for the hop duration parameter, $\delta t=4 s$, to probe short time transverse fluctuations. The probability distribution of all $R_{T}(t)$ values are shown in Fig 3D Main Text. By averaging the transverse fluctuations over all vertices, $\left\langle R_{T}(t)\right\rangle=\frac{1}{N} \sum_{i=1}^{N} R_{T}(t)_{i}$, we obtain the mean transverse fluctuation for active and passive vertices (Fig $3 C$ Main Text). $\left\langle R_{T}(t)\right\rangle$ for Cis-mutant and $\mathrm{Xdd} 1$ vertices are shown in Fig. 6F and Fig. 7F of the Main Text respectively.

Straightness Index

The directionality of the vertex trajectories were assessed using the straightness index. This is defined as the ratio of the net distance moved by a vertex between initial and final time points to the total distance moved by a vertex:

Straightness Index $=\frac{\left|\vec{r}\left(t_{f}\right)-\vec{r}\left(t_{o}\right)\right|}{\Sigma_{t}|\vec{r}(t+\delta t)-\vec{r}(t)|}$

Higher the value of the straightness index, the more directed the movement is with the value of straightness index $=1$ indicating perfectly straight line motion (see Fig. 3E, Main Text).

\section{Section 17. Perturbation of Cadherin clustering in individual junctions and its spatial periodicity}

We calculate the spatial autocorrelation of the cadherin intensity fluctuations $(C(r)$ see Section 6, Eq. (11) ) for four different embryo development scenarios, (i) wild type Cdh3 (see Fig. 4C, Main Text), (ii) Cdh3 rescue (Cdh3-GFP) (see Suppl. Fig 8A), (iii) Cdh3 cis-mutant (cisMut-Cdh3GFP) (Fig. 5D, Main Text), and (iv) Xdd1 (Fig. 7C, Main Text). The mean spatial correlation in cadherin fluctuations for wild type Cdh3 and Cdh3-GFP junctions show similar behavior with the decay to zero characterized by an exponential form (Suppl. Fig 7B). The exponential spatial dependence is evidence for the existence of a characteristic spatial scale for correlations in 
cadherin spatial distribution. The local peak in the cadherin autocorrelation function is identified using the findpeaks algorithm in MATLAB. Local peak in a data array is identified when a data point is larger than its two neighboring data points or equal to infinity. The prominence of the peak is set to 0.1 , identifying the peak amplitudes that stands out relative to other peaks. Wild type and rescue embryo cell-cell junctions are characterized by well-defined spatial periodicity in cadherin clustering, as observed from the secondary peaks in the spatial correlation (see black triangles, Suppl. Fig 7C). Therefore, cadherin spatial organization in wildtype Cdh3 and Cdh3-cis-mutant rescue embryos is in a crystal-like phase (Suppl. Fig 7C), with regularly repeating spatial patterning. Cadherin spatial correlation for individual frames (with no averaging) is shown for control (solid lines in Suppl. Fig 7D) and cadherin rescue embryos (dashed lines, Suppl. Fig 7D). However, cadherin spatial correlation in Xdd1 and cisMut-Cdh3 embryos show diffuse spatial organization, with little to no secondary peak structures visible in the spatial autocorrelation (see Suppl. Fig 7E). This is indicative of disrupted periodicity in cadherin clustering. Therefore, cadherin spatial organization in cisMut-Cdh3 and Xdd1 embryos is in a gas-like phase. Individual frame cadherin spatial correlation for cisMut-Cdh3 (solid lines Suppl. Fig 7F) and Xdd1 is shown as dashed lines in Suppl. Fig 7F. For Xdd1 and cisMut-Cdh3GFP junctions, the decay in the spatial correlation is better fit by a power law, indicating the lack of existence of a coherent length scale associated with fluctuations in cadherin expression (Suppl. Fig 7G).

\section{Section 18. Statistics}

The statistical test used and other relevant details such as the number of embryos/image frames analyzed are described in the figure legends.

\section{References}

[1] D. Thirumalai and R.D. Mountain. Activated dynamics, loss of ergodicity, and transport in supercooled liquids. Phys. Rev. E, 47(1):479 (1993).

[2] J-L Barrat, J-N Roux, and J-P Hansen. Diffusion, viscosity and structural slowing down in soft sphere alloys near the kinetic glass transition. Chem Phys, 149(1-2):197--208, (1990).

[3] T. Kirkpatrick, D. Thirumalai, Comparison between dynamical theories and metastable states in regular and glassy mean-field spin models with underlying first-order-like phase transitions. Physical Review A 37, 4439 (1988).

[4] J. Solon, A. Kaya-Copur, J. Colombelli, D. Brunner, Pulsed forces timed by a ratchet-like mechanism drive directed tissue movement during dorsal closure. Cell 137, 1331-1342 (2009). [5] A.G. Fletcher, M. Osterfield, R.E. Baker, and S.Y. Shvartsman. "Vertex models of epithelial morphogenesis." Biophysical journal 106, no. 11 (2014): 2291-2304.

[6] R.M.H Merks, M. Guravage, D. Inzé, and G.T.S Beemster. VirtualLeaf: an open-source framework for cell-based modeling of plant tissue growth and development. Plant physiology 155, no. 2 (2011): 656-666.

[7] M.F. Staddon, K.E. Cavanaugh, E.M. Munro, M.L. Gardel, S. Banerjee. Mechanosensitive junction remodelling promotes robust epithelial morphogenesis. Biophysical Journal, 117, 1-12 (2019).

[8] T. Bittig, O. Wartlick, A. Kicheva, M. González-Gaitán, and F. Jülicher. "Dynamics of anisotropic tissue growth." New Journal of Physics 10, no. 6 (2008): 063001. 
[9] P. Girard, E.A. Cavalcanti-Adam, R. Kemkemer, and J.P. Spatz. "Cellular chemomechanics at interfaces: sensing, integration and response." Soft Matter 3, no. 3 (2007): 307-326.

[10] G. Forgacs, R.A. Foty, Y. Shafrir, and M.S. Steinberg. "Viscoelastic properties of living embryonic tissues: a quantitative study." Biophysical journal 74, no. 5 (1998): 2227-2234.

[11] A. Shindo, and J.B. Wallingford. "PCP and septins compartmentalize cortical actomyosin to direct collective cell movement." Science 343, no. 6171 (2014): 649-652. 